\title{
The GRIFFIN Project-Formation of Star Clusters with Individual Massive Stars in a Simulated Dwarf Galaxy Starburst
}

\section{Lahen, Natalia}

2020-03-01

Lahen, N , Naab , T , Johansson, P H, Elmegreen, B , Hu , C-Y, Walch, S , Steinwandel, U P \& Moster , B P 2020 , ' The GRIFFIN Project-Formation of Star Clusters with Individual Massive Stars in a Simulated Dwarf Galaxy Starburst ' , Astrophysical Journal , vol. 891, no. 1 , 2 . https://doi.org/10.3847/1538-4357/ab7190

http://hdl.handle.net/10138/325490

https://doi.org/10.3847/1538-4357/ab7190

unspecified

acceptedVersion

Downloaded from Helda, University of Helsinki institutional repository.

This is an electronic reprint of the original article.

This reprint may differ from the original in pagination and typographic detail.

Please cite the original version. 
Draft Version February 19, 2020

Typeset using LATEX twocolumn style in AASTeX62

The GRIFFIN project - Formation of star clusters with individual massive stars in a simulated dwarf galaxy starburst

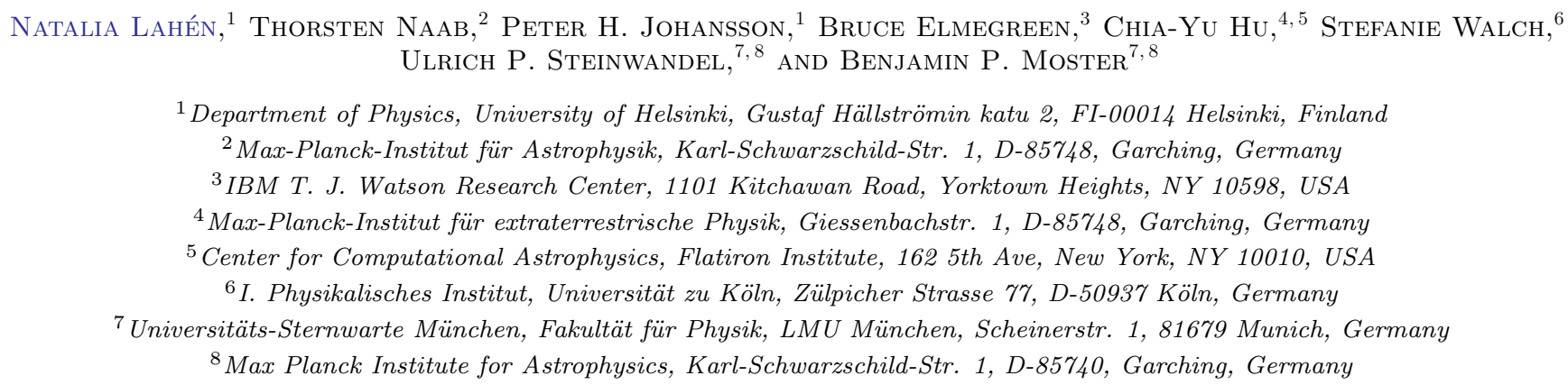

(Received 12 Nov 2019; Revised 17 Jan 2020; Accepted 28 Jan 2020)

Submitted to ApJ

\begin{abstract}
We describe a population of young star clusters (SCs) formed in a hydrodynamical simulation of a gasrich dwarf galaxy merger resolved with individual massive stars at sub-parsec spatial resolution. The simulation is part of the GRIFFIN (Galaxy Realizations Including Feedback From INdividual massive stars) project. The star formation environment during the simulation spans seven orders of magnitude in gas surface density and thermal pressure, and the global star formation rate surface density $\left(\Sigma_{\mathrm{SFR}}\right)$ varies by more than three orders of magnitude during the simulation. Young SCs more massive than $M_{*, \mathrm{cl}} \sim 10^{2.5} M_{\odot}$ form along a mass function with a power-law index $\alpha \sim-1.7(\alpha \sim-2$ for $M_{*, \mathrm{cl}} \gtrsim 10^{3} M_{\odot}$ ) at all merger phases, while the normalization and the highest SC masses (up to $\sim 10^{6} M_{\odot}$ ) correlate with $\Sigma_{\mathrm{SFR}}$. The cluster formation efficiency varies from $\Gamma \sim 20 \%$ in early merger phases to $\Gamma \sim 80 \%$ at the peak of the starburst and is compared to observations and model predictions. The massive SCs $\left(\gtrsim 10^{4} M_{\odot}\right)$ have sizes and mean surface densities similar to observed young massive SCs. Simulated lower mass clusters appear slightly more concentrated than observed. All SCs form on timescales of a few Myr and lose their gas rapidly resulting in typical stellar age spreads between $\sigma \sim 0.1-2 \operatorname{Myr}(1 \sigma)$, consistent with observations. The age spreads increase with cluster mass, with the most massive cluster $\left(\sim 10^{6} M_{\odot}\right)$ reaching a spread of 5 Myr once its hierarchical formation finishes. Our study shows that it is now feasible to investigate the SC population of entire galaxies with novel high-resolution numerical simulations.
\end{abstract}

Keywords: galaxies: dwarf — galaxies: interactions — galaxies: star clusters: general — galaxies: star formation - methods: numerical

\section{INTRODUCTION}

The majority of stars in galaxies form in hierarchical patterns (e.g. Bastian et al. 2010; Grasha et al. 2017) with dense clusters (Lada \& Lada 2003; Portegies Zwart et al. 2010) growing from sub-cluster coagulation and gas accretion on the smallest scales (McMillan et al.

Corresponding author: Natalia Lahén natalia.lahen@helsinki.fi
2007; Elmegreen 2008) and dispersing with increasing age (Baumgardt et al. 2013; Ward \& Kruijssen 2018).

As a result of this hierarchy, star clusters typically have mass functions $(\mathrm{CMF}) d N / d M \propto M^{\alpha}$ with powerlaw slopes of $\alpha \sim-2$ independent of environment (e.g. Elmegreen \& Falgarone 1996; Elmegreen \& Efremov 1996; Zhang \& Fall 1999; Hunter et al. 2003; Parker \& Goodwin 2007; Fall \& Chandar 2012). The lower cluster mass limit of $\sim 10^{2} M_{\odot}$ is set by the masses of individual stars, while some authors find evidence for an extended 
power-law distribution of clusters all the way to very high masses of $10^{6} M_{\odot} \lesssim M_{*} \lesssim 10^{7} M_{\odot}$ (e.g. Mok et al. 2019). In the case of the extended power-law CMF, globular clusters with typical masses of $M_{*} \sim 10^{5} M_{\odot}$ would just be the massive relics of normal star clusters forming in extreme environments at high redshift (Elmegreen \& Efremov 1997; Fall \& Zhang 2001; Kruijssen 2015; Vanzella et al. 2019). On the other hand, several studies imply an upper cutoff around $M_{*} \sim 10^{5} M_{\odot}$ (Portegies Zwart et al. 2010; Adamo et al. 2015; Messa et al. 2018a), making the CMF resemble a Schechter function, which is described by a power-law with an exponential tail.

In the local Universe the formation of star clusters is enhanced in extreme environments, such as galaxy mergers, starburst galaxies and galactic nuclei (e.g. Meurer et al. 1995; Whitmore et al. 1999; Bastian et al. 2005; Genzel et al. 2010; Pfeffer et al. 2019). It is debated in the observational literature, whether the cluster formation rate follows the global star formation rate, independent of galaxy type (e.g. Chandar et al. 2015, 2017) or if the efficiency for young clusters surviving the embedded phase increases for galaxies with higher star formation rates (e.g. Goddard et al. 2010; Kruijssen 2012; Pfeffer et al. 2019). In the first case, the fraction of young stars that are in clusters is independent of the star formation rate per unit area, whereas in the second case, the fraction increases with the star formation rate per unit area.

The formation of the population of young massive clusters (YMC) is of particular interest. They are very compact, with half-light radii of only a few pc and masses of $\sim 10^{4}-10^{8} M_{\odot}$, which is similar to the masses of present-day globular clusters (e.g. Larsen 2004; Portegies Zwart et al. 2010; Rochau et al. 2010; Cottaar et al. 2012). Given their many similarities, many authors have suggested that the present-day globular clusters are just YMCs formed at early times (Longmore et al. 2014; Kruijssen 2014). GCs would then be the fossil record of an intense early episode of clustered star formation, which is evidenced in the present-day population of globular clusters found ubiquitously in the haloes of all types of galaxies, even in low-mass dwarf galaxies (e.g. Harris 1991; Bastian \& Lardo 2018).

The observations of YMCs demonstrate that they are preferentially formed in very dense and gas-rich environments characterized by strongly turbulent velocity fields and very high gas pressures of $P_{\mathrm{th}} \gtrsim 10^{7} \mathrm{k}_{\mathrm{B}}\left(\mathrm{K} \mathrm{cm}^{-3}\right)^{-1}$. Such high densities and pressures, uncommon in the local Universe but more prevalent in the high redshift Universe, also result in elevated integrated star formation efficiencies, in which a larger fraction of the mass of a given molecular cloud might be turned into stars (e.g. Fall et al. 2010; Skinner \& Ostriker 2015; Li et al. 2019). The increased star formation efficiency in turn would also result in a higher cluster formation efficiency (CFE), which describes the fraction of star formation occurring in bound stellar clusters (e.g. Elmegreen \& Efremov 1997; Kruijssen 2012). Even if the cluster formation efficiency is independent of environment, higher gas surface densities and therefore star formation rate surfaces densities would result in a higher normalization of the CMF and therefore an increase in the peak cluster mass (e.g. Whitmore et al. 2014). Thus, a mechanism that can generate high gas densities, such as a galaxy merger, will be most beneficial for studying a large population of stellar clusters.

Traditionally, star cluster evolution has been investigated using direct $N$-body simulations that resolve the internal evolution of pre-existing star clusters set in a tidal field at high spatial and temporal precision (McMillan et al. 2007; Renaud et al. 2011; Wang et al. 2016). However, the direct $N$-body simulations typically do not include hydrodynamical processes, which are crucial for studying the actual formation process of stellar clusters as stars form in dense molecular clouds. In addition, due to the steep scaling of computational time $O\left(N^{2}\right)$ with particle number $N$, these simulations are often unable to model the environment around the stellar clusters and therefore lack the detailed interplay between the clusters and their host galaxy.

To date, most of the galactic-scale numerical simulation work has concentrated on simulating the formation of massive clusters $\left(>10^{3} M_{\odot}\right)$ such as proto-globular clusters (Renaud et al. 2015; Kim et al. 2018; Ma et al. 2019; Renaud 2018) with the stars represented as population particles resolved down to $10^{2} M_{\odot}$ (see e.g. Hu et al. 2017; Emerick et al. 2018, for recent improvements towards realizations of individual massive stars). Observations in the Milky Way probe cluster masses down to a minimum mass of only a few tens of solar masses (Lada \& Lada 2003; van den Bergh 2006). One of the major challenges in modelling the formation of stellar clusters in a realistic galactic environment are their very compact sizes. The effective radii of clusters are typically in the range of only $\sim 1-10$ parsecs (O'Connell et al. 1994; McLaughlin \& van der Marel 2005; Bastian et al. 2013), thus resolving their formation process will require high spatial resolution.

Numerically the formation of stellar clusters has been studied using isolated simulations of collapsing and fragmenting molecular clouds (e.g. Klessen 2001; Bonnell et al. 2003; Mapelli 2017; Padoan et al. 2017, VázquezSemadeni et al. 2017; Howard et al. 2018). In addition, 
the properties of star clusters have also been studied in stratified disk models (e.g. Walch et al. 2015; Peters et al. 2017; Kim \& Ostriker 2018), in spiral galaxies and their mergers (e.g. Bekki et al. 2002; Li et al. 2004, 2005; Bournaud et al. 2008) and in dwarf galaxies (e.g. Saitoh et al. 2010; Hopkins et al. 2013), however typically without resolving their internal structure. Idealised merger simulations provide a high spatial and mass resolution (Renaud et al. 2015; Arata et al. 2018), even down to solar masses per resolution element (Lahén et al. 2019), but lack the cosmological environment provided by cosmological simulations. Meanwhile current cosmological zoom-in simulations have been used to gain insight in the formation of stellar clusters. However, the highest available mass resolution is still of the order of hundreds of solar masses ( $\mathrm{Ma}$ et al. 2019). Interpreting the formation of stellar clusters in a full cosmological context is also possible in a statistical sense (Renaud et al. 2017; Li et al. 2017; Choksi et al. 2018; Choksi \& Gnedin 2019; El-Badry et al. 2019; Halbesma et al. 2019) or by including additional semi-analytical modelling (Bekki et al. 2008; Pfeffer et al. 2018). Recent analytical models favor a "conveyor belt" model (Longmore et al. 2014) with gas accretion and star formation happening simultaneously (Krumholz \& McKee 2019) which is supported by the analysis of the most massive clusters forming in our simulation (Lahén et al. 2019).

In this paper we present a dwarf galaxy merger simulation with gas-rich initial conditions, which provide an ideal environment for efficient star and cluster formation. Crucially, the simulation has a minimum gas particle mass of $4 M_{\odot}$ and realizes individual massive stars with their individual tracks and models their radiation and supernova feedback at sub-parsec spatial resolution. This enables us to study the formation and evolution of the stellar cluster population from globular cluster masses down to almost the smallest observed cluster masses of $\sim 200 M_{\odot}$ in a starbursting galactic environment. In an earlier study Lahén et al. (2019, L19 hereafter) we studied the properties of massive, globular cluster-like objects, which populate the high-mass end of the cluster mass function. In the present study we concentrate on describing the entire young cluster population formed during the merger and show that our simulated clusters form rapidly on timescales of a few Myrs with properties similar to the observed local cluster population.

The simulation is a part of the GRIFFIN project, which is an acronym for Galaxy Realizations Including Feedback From INdividual massive stars. The aim of this project is to perform galaxy scale simulations of individual galaxies, galaxy mergers, and cosmological zoom simulations at such high resolution and physical fidelity that individual massive stars can be realised and important feedback processes such as supernova explosions (Steinwandel et al. 2019) can be reliably included to study the formation of a realistic non-equilibrium multiphase interstellar medium (Hu et al. 2017). This level of detail in modern simulations is very important as the environmental density of supernova explosions is controlled by stellar feedback processes and to a large extent by stellar clustering (see Naab \& Ostriker 2017, for a detailed discussion of this challenge). A numerical model reliably representing the fundamental mass unit of single massive stars and the fundamental energy injection unit of individual supernova explosions in realistic star cluster populations provides unique insights into the physical mechanisms regulating the multi-phase structure of the galactic ISM as well as the driving of galactic outflows - and therefore galaxy evolution as a whole (Naab \& Ostriker 2017). In this paper we use the high dynamic fidelity of a dwarf merger simulation to study the formation of galactic populations of star clusters across environments changing by many orders of magnitude in density. We show that our model, which has a self-consistently evolving multi-phase interstellar medium, produces CMFs similar to observations over four orders of magnitude in star cluster masses with the most massive systems being realistic proto-globular clusters (Lahén et al. 2019).

This article is organized as follows. The main aspects of the simulation code and initial conditions are described in Section 2. A general overview of the simulation, including the star formation history is presented in Section 3. This section also introduces our stellar cluster identification procedure and discusses the cluster formation during the simulation. The properties of the young cluster population are analysed in detail in Section 4 , with the results also being compared to the locally observed stellar cluster population. Finally, we present our conclusions in Section 5.

\section{SIMULATIONS}

\subsection{The simulation code}

The simulations were run with a modified version of the well-tested smoothed particle hydrodynamics (SPH, Lucy 1977; Gingold \& Monaghan 1977) tree code GADGET-3 (Springel 2005). Gas dynamics is modelled with the SPH implementation SPHGAL, presented in Hu et al. (2014, 2016, 2017), using the pressure-energy formulation (Read et al. 2010; Hopkins 2013; Saitoh \& Makino 2013). The gas properties are smoothed over 100 neighboring particles using the Wendland $C^{4}$ kernel (Dehnen \& Aly 2012). To stabilize the SPH scheme, we 
also model artificial viscosity (Cullen \& Dehnen 2010) with a few important modifications (see Hu et al. 2014) and artificial conduction of thermal energy (Price 2008; Read et al. 2010) in converging gas flows. The time stepping is regulated with a limiter which keeps neighboring particles within a time step difference by a factor of four to capture shocks accurately. All technical details are given in $\mathrm{Hu}$ et al. $(2014,2016,2017)$. We briefly review the implementations including updates to the star formation model in section 2.3.

\subsection{Chemistry and cooling}

We track the chemical composition of gas and stars by following the abundance of 12 elements $(\mathrm{H}, \mathrm{He}, \mathrm{N}, \mathrm{C}, \mathrm{O}$, $\mathrm{Si}, \mathrm{Mg}, \mathrm{Fe}, \mathrm{S}, \mathrm{Ca}, \mathrm{Ne}$ and $\mathrm{Zn}$ ) and six chemical species $\left(\mathrm{H}_{2}, \mathrm{H}^{+}, \mathrm{H}, \mathrm{CO}, \mathrm{C}^{+}, \mathrm{O}\right)$ as well as the free electron density. Dust, which constitutes $0.1 \%$ of the gaseous mass at the adopted $\sim 0.1 Z_{\odot}$ metallicity, contributes to $\mathrm{H}_{2}$ formation and also the shielding of radiation in the gas clouds. Dust is assumed to be in thermal equilibrium where the dust temperature is calculated from the balance of cooling and heating as described in Glover \& Clark (2012). The dust heating processes included here are stellar emission (the interstellar radiation field), $\mathrm{H}_{2}$ formation on dust, and dust-gas collisions.

The cooling rates for gas are modelled in two temperature regimes. Below $T=3 \times 10^{4} \mathrm{~K}$ we use the chemical network described in detail in $\mathrm{Hu}$ et al. (2016), based on Nelson \& Langer (1997), Glover \& Mac Low (2007) and Glover \& Clark (2012), to follow low-temperature cooling down to $T=10 \mathrm{~K}$. High-temperature cooling above $T=3 \times 10^{4} \mathrm{~K}$ is modelled using the metallicitydependent cooling tables from Wiersma et al. (2009) assuming an optically thin ISM in an ionizing UVbackground from Haardt \& Madau (1996).

\subsection{Star formation}

The onset of gravitational collapse for gas at the local SPH averaged density $\rho_{i}$ can be approximated with the local Jeans mass, defined as

$$
M_{J, i}=\frac{\pi^{5 / 2} c_{s, i}^{3}}{6 G^{3 / 2} \rho_{i}^{1 / 2}}
$$

where $c_{s, i}$ is the local sound speed and $G$ is the gravitational constant. The gas is allowed to form stars stochastically at star formation (SF) efficiency $\epsilon_{\mathrm{SF}}=2 \%$ if the local Jeans mass is less than $8 \mathrm{SPH}$ kernel masses, which corresponds to $\sim 3200 M_{\odot}$. We also require a gas particle to be in a converging flow to form stars. The probability for a gas particle, which meets these criteria, to turn into a stellar particle is then set as $1-\exp (-p)=1-\exp \left(-\epsilon_{\mathrm{SF}} \Delta t / t_{\mathrm{ff}}\right)$, where $t_{\mathrm{ff}}$ is the local free-fall time $t_{\mathrm{ff}} \sim\left(4 \pi G \rho_{i}\right)^{-1 / 2}$ given by the SPH quantities, and $\Delta t$ is the length of the time-step (Hu et al. 2017).

In addition to $\mathrm{Hu}$ et al. (2017), we enforce star formation if the Jeans mass is resolved by less than 0.5 SPH kernel masses $\left(\sim 200 M_{\odot}\right)$. Any gas particle ending their time step below this threshold is instantaneously turned into a star particle, ignoring the converging flow criterion. The instantaneous star formation limit corresponds to hydrogen number densities of $n_{\mathrm{H}} \gtrsim 10^{3.5} \mathrm{~cm}^{-3}$ and temperatures of $T=10-100 \mathrm{~K}$. For phase diagrams illustrating the gas properties and the SF thresholds, see Fig. 1 in L19.

\subsection{IMF sampling}

We sample the mass of a star-forming gas particle into an array of stellar masses by drawing randomly from a Kroupa initial mass function (IMF) (Kroupa 2001). This enables us to resolve massive stars with individual stellar particles: gas particles which draw a stellar mass greater than the gas mass resolution of $\sim 4 M_{\odot}$ represent individual massive stars. Lower mass stars are stored as stellar populations with total mass equal to the mass of the original gas particle. Whenever the total sampled mass exceeds the mass of the original gas particle, the exceeding mass is reduced from the next star forming gas particles in order to conserve mass. Each new stellar particle also inherits the chemical composition of their progenitor gas particle.

\subsection{The Interstellar radiation field}

We model the spatially and temporally evolving interstellar radiation field (ISRF) emanating from stellar particles which include sampled stars younger than their lifetimes. The mass-dependent stellar lifetimes are calculated from Georgy et al. (2013) assuming a metallicity of $Z=0.002 \approx 0.1 Z_{\odot}$. The stellar particles emit a far-ultraviolet (FUV) radiation field out to a radius of 50 pc for which we assume an optically thin medium, appropriate for our low-metallicity system. The stellar FUV spectrum is integrated from the BASEL library (Lejeune et al. 1997, 1998; Westera et al. 2002) in the range 6-13.6 eV, and summed up for all (young enough) stars in each stellar particle. We account for dust extinction and shielding by the chemical species using the TreeCol (Clark et al. 2012) algorithm along 12 line-ofsight columns obtained using HEALPIX (Górski \& Hivon 2011).

We also propagate the hydrogen-ionizing radiation from massive stars assuming a balance between the production rate of ionizing photons and the recombination rate in the ISM surrounding the stars. The photoionization implementation produces successfully analytical 

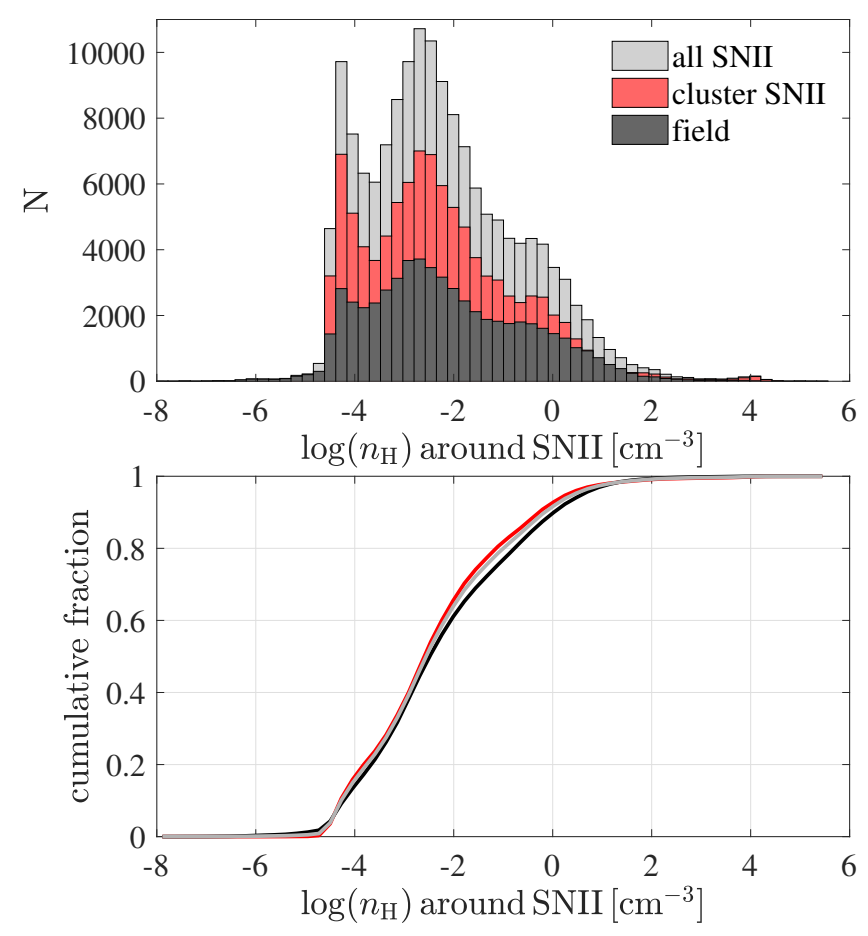

Figure 1. Distribution of all environmental densities of SNII during the simulation up to $100 \mathrm{Myr}$ after the starburst. The SNII events have been separated according to whether the SN remnant is a star cluster member. About $97 \%$ of the SNII explode at densities $\lesssim 10 \mathrm{~cm}^{-3}$ and can be considered well resolved at this resolution. Less than $1 \%$ of the SN explode at densities of $\gtrsim 100 \mathrm{~cm}^{-3}$ and have marginally or un-resolved hot phase generation. Still, the radial momentum injection is typically captured (Hu et al. 2016; Steinwandel et al. 2019).

solutions to D-type expansion fronts (Spitzer 1978; Bisbas et al. 2015) and it can cope with overlapping HII regions around young massive stars. The impact of the ionizing radiation on the ISM is important for the accurate modelling of the following supernova events, as the massive stars heat up their surroundings before they explode in a lower-density medium, as opposed to the stellar birth cloud (Gatto et al. 2015).

\subsection{Stellar feedback}

Each individual star with a mass greater than $8 M_{\odot}$ will explode as a type II supernova (SNII) at the end of its stellar lifetime (Georgy et al. 2013). At our mass resolution we resolve the expansion of the supernova remnants self-consistently with stochastic energy injection (see Appendix B in Hu et al. 2016), where each SNII releases $10^{51}$ erg as thermal energy into 100 nearest gas particles weighted by a cubic spline kernel. In addition, the SNII events release metals according to the metallicity-dependent ejecta release rates obtained from Chieffi \& Limongi (2004) at an ejection velocity of 3000 $\mathrm{km} / \mathrm{s}$. Stars also release AGB (asymptotic giant branch) winds at a more gradual rate and lower velocity of 25 $\mathrm{km} / \mathrm{s}$, with metal yields obtained from Karakas (2010).

Because of stellar motion out of the dense birth places, stellar clustering, and photoionization (Hu et al. 2017), the vast majority of the SNII events go off in a considerably less dense gaseous environment than e.g. the densities at which the star clusters form (see e.g. Girichidis et al. 2016; Peters et al. 2017; Hu et al. 2017; Naab \& Ostriker 2017). In Fig. 1 we show a histogram of the ISM densities around all SNII events that occur until $100 \mathrm{Myr}$ past the starburst. In the top panel of Fig. 1 we separate the supernova remnants which become part of star clusters (see Section 3.4) and the field remnants, and show the cumulative distribution in the bottom panel. Over $97 \%$ of the SNII explode at ISM number densities less than $n_{\mathrm{H}}=10 \mathrm{~cm}^{-3}$ and can be considered well resolved (Hu et al. 2016). Only $1 \%$ of all the SNII explode at a density above $n_{\mathrm{H}}=100 \mathrm{~cm}^{-3}$, out of which more than $70 \%$ occur in clusters. The peak of the density distribution is at $\sim 10^{-2} \mathrm{~cm}^{-3}$ similar to other high resolution simulations with structured multi-phase ISM properties (Girichidis et al. 2016; Peters et al. 2017; Hu et al. 2017).

The kernel weighting of the feedback ejecta favors the nearest particles, and at times single gas particles may receive up to a few times their original mass worth of stellar material when for example a nearby massive star explodes as a type II supernova. Whenever a gas particle exceeds a mass limit of twice the original gas mass resolution (here $4 M_{\odot}$ ), its mass is split into two new particles. The ability of the code to resolve supernova blast waves in different environments has been tested in detail in Hu et al. (2017) and Steinwandel et al. (2019).

\subsection{Initial conditions}

The initial conditions are based on the dwarf galaxy model used in the simulation studies performed in $\mathrm{Hu}$ et al. $(2016,2017)$. The two identical dwarf galaxies with virial masses of $M_{\text {vir }}=2 \times 10^{10} M_{\odot}$ and virial radii of $r_{\text {vir }}=44 \mathrm{kpc}$ are both composed of a dark matter halo and a gas-rich disk with a rotationally supported stellar population. The dark matter halo is represented with a Hernquist density profile (Hernquist 1990) with an NFW-equivalent concentration parameter of $c=10$ (Springel et al. 2005) and spin parameter of $\lambda=0.03$ (Bullock et al. 2001).

The exponential stellar disk with a mass of $M_{*}=2 \times$ $10^{7} M_{\odot}$ is set up with scale radius of $r_{*}=0.73 \mathrm{kpc}$ and scale height of $h_{*}=0.35 \mathrm{kpc}$. The gaseous disk has initially a mass of $M_{\text {gas }}=4 \times 10^{7} M_{\odot}$ and scale 


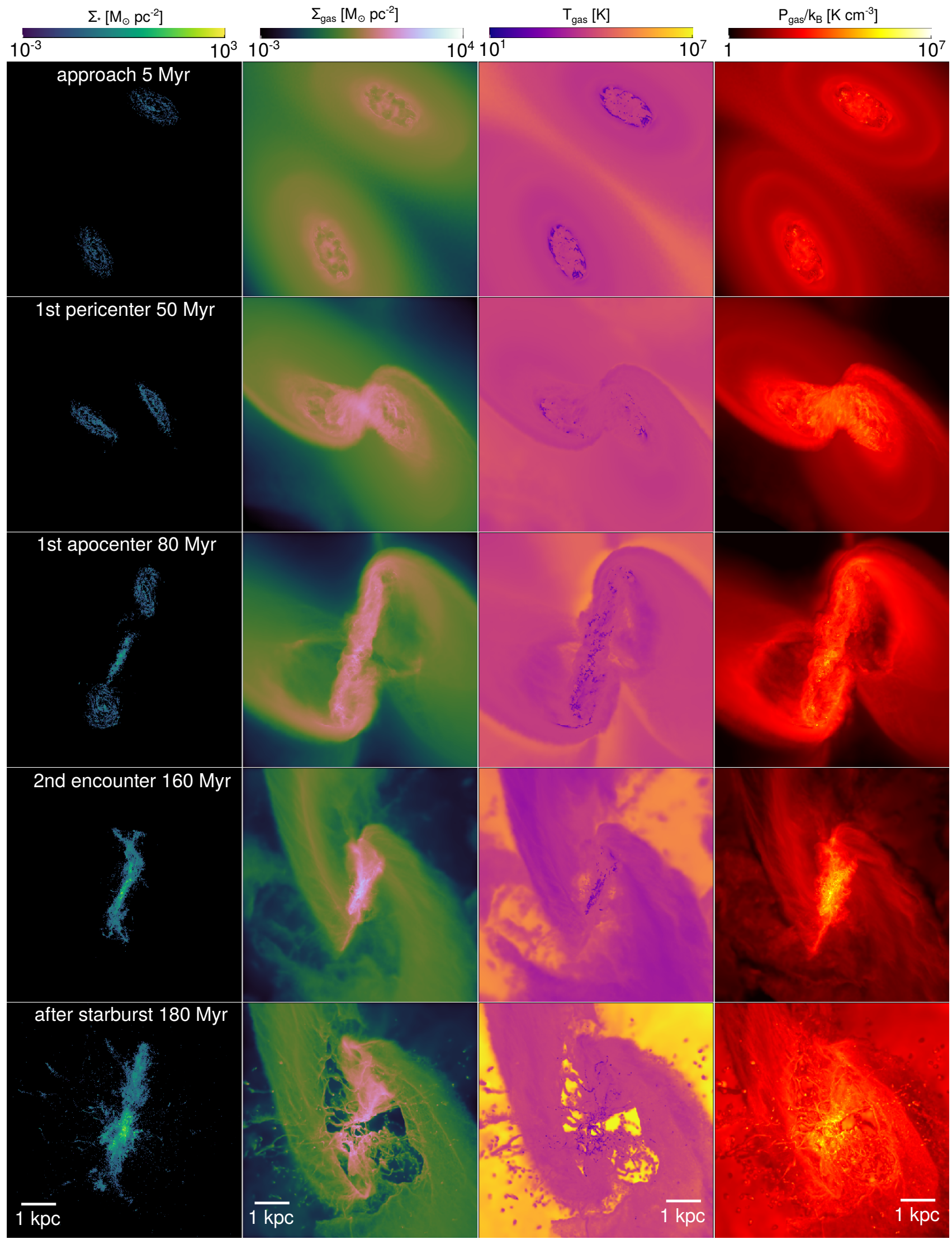

Figure 2. An overview of the merger. The rows from top to bottom depict a projection in the $x-y$ plane at the approach, the first pericentric passage, the first apocenter, the onset of the starburst, and the interaction slightly after the starburst. The columns from left to right show the surface density of stars formed during the simulation, the surface density of gas, the density weighted gas temperature, and the density weighted thermal gas pressure. The panels span a $(7 \mathrm{kpc})^{3} \mathrm{box}$. 
radius twice that of the stellar disk, $r_{\text {gas }}=1.46 \mathrm{kpc}$, and the vertical structure is calculated assuming hydrostatic equilibrium. The gaseous metallicity is initially set to $Z_{\text {gas }}=0.002 \approx 0.1 Z_{\odot}$, which is a typical value for lowmass dwarf galaxies.

Each dwarf galaxy consists of 4 million dark matter particles, 10 million gas particles and 5 million stellar particles, defining a particle mass resolution of $m_{\mathrm{DM}}=$ $7 \times 10^{3} M_{\odot}$ and $m_{\text {bar }}=4 M_{\odot}$ for the dark matter and baryonic components, respectively. The gravitational softening lengths are set to $\epsilon_{\mathrm{DM}}=62 \mathrm{pc}$ for dark matter and $\epsilon_{\mathrm{bar}}=0.1 \mathrm{pc}$ for baryonic particles. The two dwarf galaxies are set on parabolic orbits with a pericentric distance of $d_{\text {peri }}=1.46 \mathrm{kpc}$ and an initial separation of $d_{\text {init }}=5 \mathrm{kpc}$, leading to a fairly rapid coalescence time for the gas disks. The inclination and argument of pericenter are chosen off-plane as $\left\{i_{1}, i_{2}\right\}=\left\{60^{\circ}, 60^{\circ}\right\}$ and $\left\{\omega_{1}, \omega_{2}\right\}=\left\{30^{\circ}, 60^{\circ}\right\}$. We chose the same initial orientations as for the Antennae-like simulations studied in Lahén et al. (2018).

As presented in L19, we have run the simulation for up to $100 \mathrm{Myr}$ after the merger, for a total of $\sim 300 \mathrm{Myr}$. Here we concentrate on the first $200 \mathrm{Myr}$ of the simulation time, up to some $40 \mathrm{Myr}$ past the starburst. To assess the effects specific to the merging dwarf galaxies, we also run one dwarf galaxy in isolation for the same total simulation duration. More detailed discussion on the properties of the isolated dwarf can be found in $\mathrm{Hu}$ et al. $(2016,2017)$.

\section{STAR FORMATION IN A GAS-RICH DWARF GALAXY MERGER}

\subsection{Simulation overview}

A general overview of the evolution of the stellar and gaseous components is shown in Fig. 2 for five epochs during the simulation: the approach at $5 \mathrm{Myr}$, the first pericentric passage at $50 \mathrm{Myr}$, the first apocenter at 80 Myr, the second encounter and the onset of the starburst at $160 \mathrm{Myr}$, and at $180 \mathrm{Myr}$, slightly past the most intense starburst.

The dwarfs begin to show signs of the interaction first in the outskirts of their extended gas disks. The interaction-induced gas compression is already strong further away from the galactic centers, thus the distribution of star formation is relatively more extended compared to the interactions between more massive latetype galaxies that show more centrally concentrated star formation (e.g. Sanders \& Mirabel 1996; Smith et al. 2007; Ellison et al. 2013). During the first encounter and up to a simulation time of $100 \mathrm{Myr}$, the tidal bridge dominates the star formation over the combined dwarf disks (see the third row in Fig. 2). During the second pas- sage and the starburst phase there are multiple clumpy star formation regions which are distributed mostly in a region $2 \mathrm{kpc}$ across, comparable to the scale radii of the initial disks. Most of the star formation is also located half a kiloparsec off-center from the dark matter distribution. When the SFR peaks, the gas disks are settling into the central region, where the most massive stellar clusters are forming. After the starburst, the supernova feedback from the stellar clusters unbinds the gas even in the densest star-forming regions, dispersing the gas and halting the most intense starburst.

\subsection{The global star formation rate}

The total SFR increases to a few times $10^{-3} M_{\odot} \mathrm{yr}^{-1}$ during the first pericentric passage. During the starburst, the SFR peaks at $\sim 0.2-0.3 M_{\odot} \mathrm{yr}^{-1}$ at a simulation time of $t=160-170 \mathrm{Myr}$, which corresponds to a specific SFR of $\mathrm{sSFR}=4.6-7 \times 10^{-9} \mathrm{yr}^{-1}$ at the total stellar mass of $\sim 4.3 \times 10^{7} M_{\odot}$. At its highest, the specific SFR is therefore up to an order of magnitude above the general star-forming main sequence (Salim et al. 2007; Schiminovich et al. 2007; Huang et al. 2012), although the observed scatter in the relation is quite large for low mass galaxies (Bothwell et al. 2009; Pearson et al. 2016). The SFR in our merger is similar to interacting dwarf galaxies in general (Pearson et al. 2016; Paudel et al. 2018). In the context of other numerical studies, for example the dwarf merger system presented in Privon et al. (2017) has a stellar mass of $\sim 9 \times 10^{7} M_{\odot}$ and a total SFR of a few $0.1 M_{\odot} \mathrm{yr}^{-1}$.

The total star formation efficiency, calculated as all stellar mass formed during the simulation divided by the original gas mass, reaches $6.3 \%$ by the end of the starburst. With respect to the pre-existing stellar disk, the newly formed stars account for $11 \%$ of all stellar matter in the system.

\subsection{Resolved $\Sigma_{\mathrm{SFR}}-\Sigma_{\text {gas }}$-relation}

The evolution of the star formation rate surface density $\Sigma_{\mathrm{SFR}}$ with respect to gas surface density $\Sigma_{\text {gas }}$ is shown in Fig. 3 in 1 Myr steps for both the merger and the isolated dwarf galaxy. The evolution along the merger is color coded by time. We calculate the resolved values by default in $400 \mathrm{pc}$ by $400 \mathrm{pc}$ pixels, and only include in our calculation pixels with ongoing star formation, akin to the SFR weighted $\Sigma_{\mathrm{SFR}}$ in e.g. Johnson et al. (2017).

We also model the effects of observational restrictions by calculating the $\Sigma_{\mathrm{SFR}}$ and $\Sigma_{\text {gas }}$ values from both the full dataset and by applying the observational sensitivity limits of the THINGS survey (Leroy et al. 2008), which provides spatially resolved measurements at sub- 


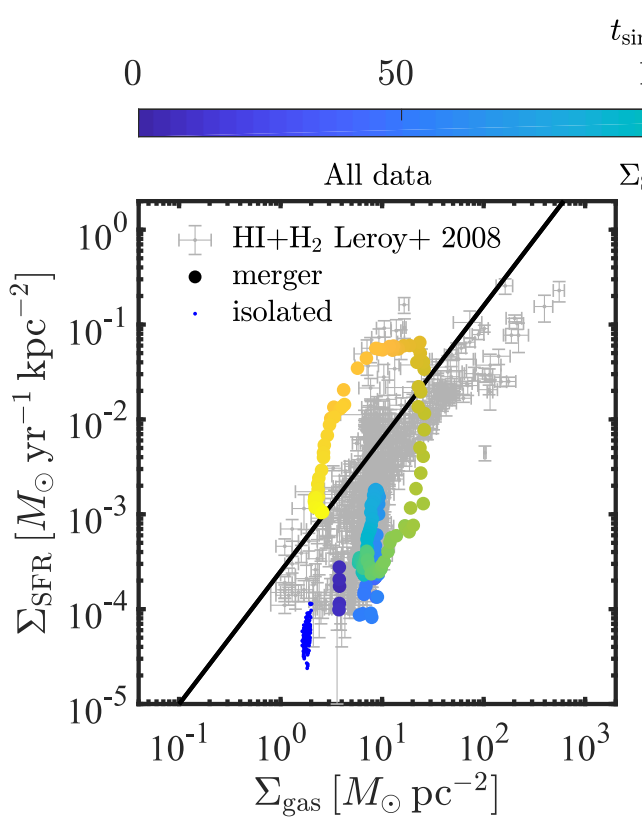

$t_{\text {sim }}[\mathrm{Myr}]$

100

150

200
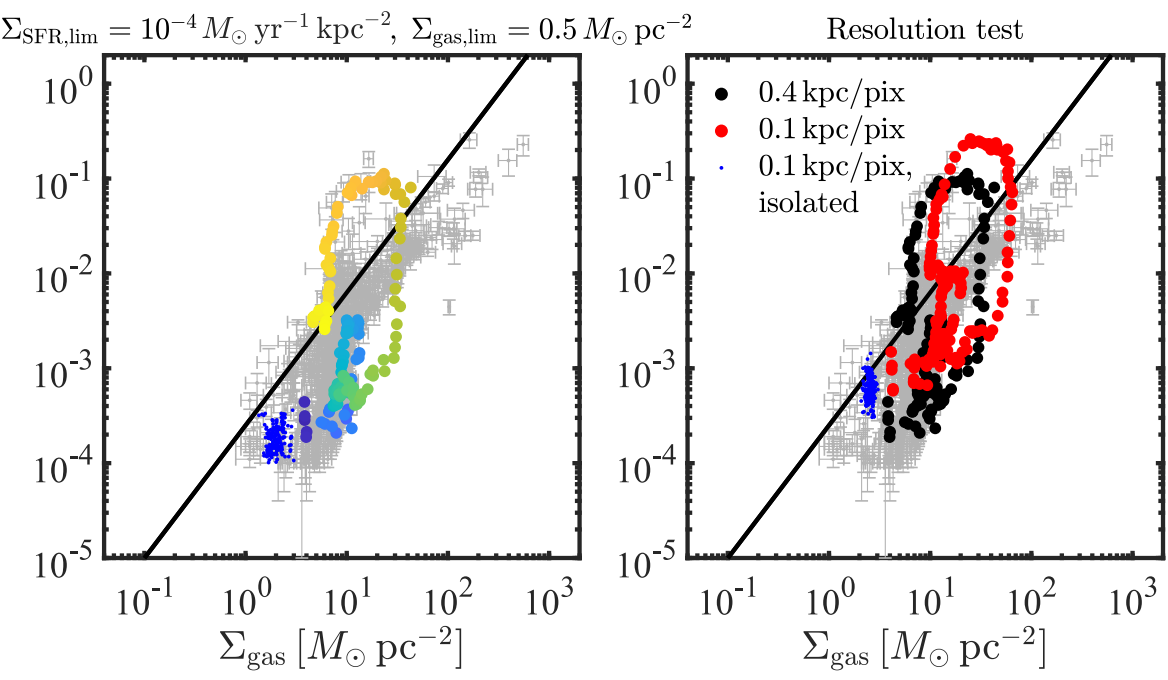

Figure 3. The evolution of the projected SF properties in the merger and the isolated galaxy. The leftmost panel shows the full dataset without any observational restrictions averaged over pixels with SF, at a resolution of 400 pc per pixel. In the middle panel the average over gas surface density and SFR surface density have been limited to pixels exceeding the observational sensitivity limits of $\Sigma_{\text {gas,lim }}=0.5 M_{\odot} \mathrm{pc}^{-2}$ and $\Sigma_{\mathrm{SFR} \text {,lim }}=10^{-4} M_{\odot} \mathrm{yr}^{-1} \mathrm{kpc}^{-2}$, analogous to the sensitivity in the THINGS survey (Leroy et al. 2008). The middle and right panels additionally only consider pixels including IMF sampled stars above $8 M_{\odot}$, to mimic observing UV and $\mathrm{H}_{\alpha}$ emission. The rightmost panel shows a resolution study for the data in the middle panel. Observed galaxies from Leroy et al. (2008) are shown on the background and the diagonal line shows the traditional Kennicutt-Schmidt (Kennicutt 1998) relation of $\boldsymbol{\Sigma}_{\mathrm{SFR}}=\mathbf{2 . 5} \times \mathbf{1 0}^{-\mathbf{4}} \boldsymbol{\Sigma}_{\text {gas }}^{\mathbf{1 . 4}}$.

kpc resolution. Firstly, we only include pixels with stellar particles where the IMF sampling has produced a star more massive than $8 M_{\odot}$ when we calculate the SFR surface density. This mimics observing the far-UV and re-emitted $24 \mu \mathrm{m}$ dust emission originating from $\mathrm{O} / \mathrm{B}$ stars. Secondly, we apply sensitivity limits to both the SFR surface density, $\Sigma_{\mathrm{SFR}, \lim }=10^{-4} M_{\odot} \mathrm{kpc}^{-2}$, and the gas surface density, $\Sigma_{\text {gas,lim }}=0.5 M_{\odot} \mathrm{pc}^{-2}$, which ignore the regions with too weak signal to be detected. The observational limits reduce the number of detected pixels from the typical values of $50-200$ by $30-60 \%$ during the first and second passage and the starburst phase, and by up to $\sim 70 \%$ thereafter.

The leftmost panel of Fig. 3 shows the relationship between the gas density and star formation rate surface density without the application of any observational limitations, whereas the middle and right side panels include the observational restrictions detailed above. The final result for each snapshot is an average over all the pixels with a detectable signal. We also test the effect of better image resolution in the rightmost panel of Fig. 3. The obtained values are compared to the global Kennicutt-Schmidt star formation relation of the form $\Sigma_{\mathrm{SFR}} \propto \Sigma_{\text {gas }}^{1.4}$ (Kennicutt 1998) and the observed $\Sigma_{\mathrm{SFR}^{-}}$ $\Sigma_{\text {gas }}$ values from the THINGS survey (Leroy et al. 2008) which has a pixel resolution of $400 \mathrm{pc} / \mathrm{pix}$ in dwarfs and $800 \mathrm{pc} / \mathrm{pix}$ in other galaxies.

The isolated dwarf is located in the low $\Sigma_{\mathrm{SFR}}-\Sigma_{\text {gas }}$ region of the observed dataset, as could be expected for a quiescent dwarf galaxy. In the middle panel of Fig. 3 the mean $\Sigma_{\mathrm{SFR}}$ increases to the imposed $\Sigma_{\mathrm{SFR}}$ limit when pixels with only a few new stars and therefore not enough signal, are ignored. The gas distribution is fairly uniform at $400 \mathrm{pc}$ per pixel scale, therefore the limit on $\Sigma_{\text {gas }}$ does not affect the mean results for $\Sigma_{\text {gas }}$. For a resolved, more detailed study of the star formation properties of the isolated dwarf, see Hu et al. (2016).

The merger starts from the same region as the isolated dwarf, and evolves through most of the entire range of observed data during the simulation. The observational restrictions in the middle panel shift the simulated data slightly up along the traditional KS-relation, as for example blown out gas and small isolated star formation regions are excluded from the global mean. The poststarburst data points of the merger shift to higher gas surface densities because the clumpy star formation produces high-mass stars more frequently in high-density regions, which are less severely affected by the observational restrictions imposed in the middle panel of Fig. 3. 


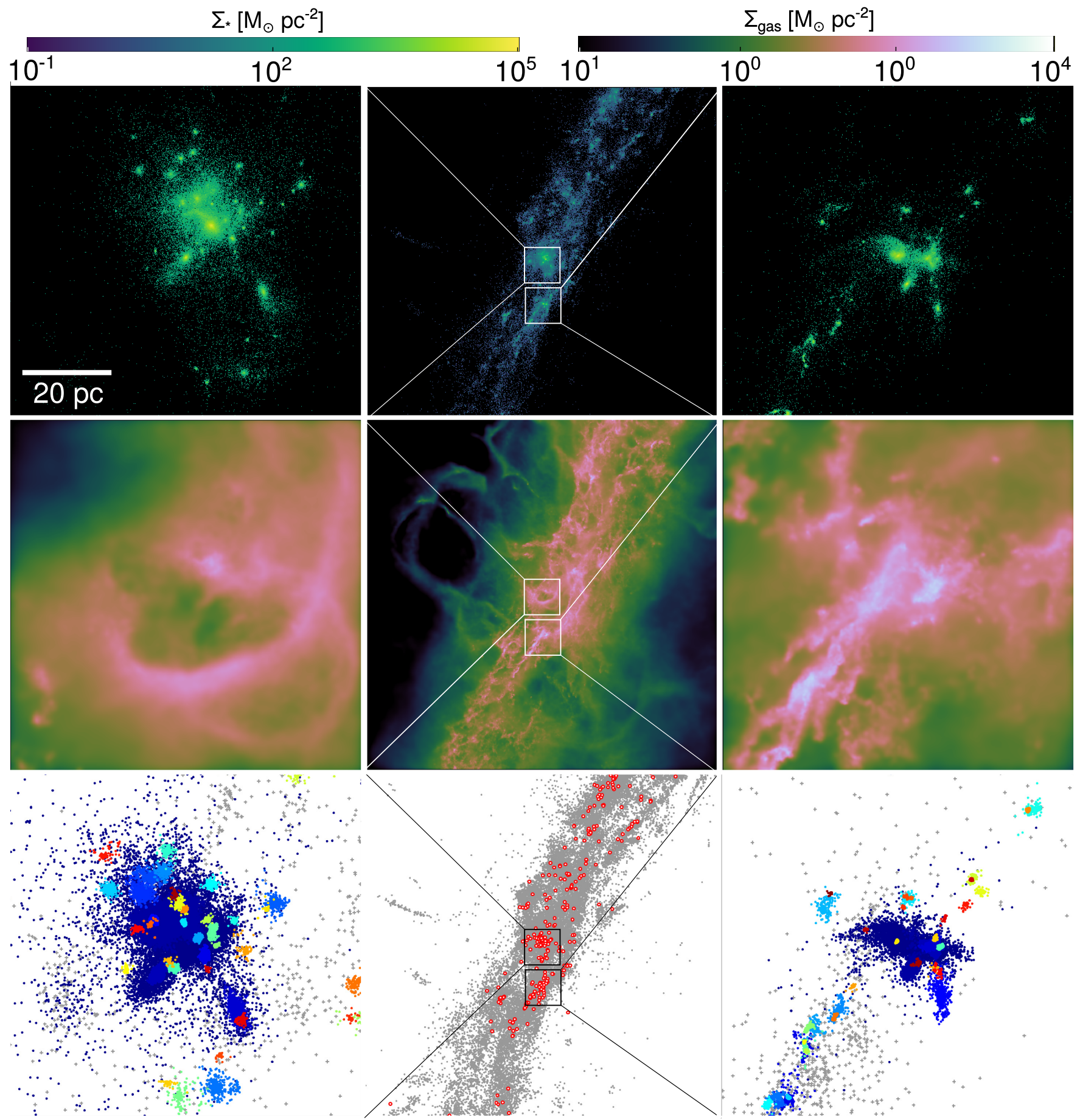

Figure 4. An $x-y$ projection of the stellar and gaseous structure around the central cluster formation region at a simulation time of $t \sim 160 \mathrm{Myr}$, before the first massive cluster blows out the super-bubble. The top and middle rows show the stellar surface density and gas surface density around the two most massive star formation region at that time. The left and right bottom panels show the bound cluster particles (colored points) and unbound stellar particles (gray) within the respective regions. All cluster positions are shown with red points in the middle panel of the bottom row. The left and right columns show 80 pc by 80 pc by 80 pc zoomed slices of the regions shown in the middle column, which spans a 800 pc by 800 pc by 800 pc slice centered at the first forming massive cluster. 
The rightmost panel of Fig. 3 shows for comparison the data from the middle panel calculated at a resolution of $100 \mathrm{pc}$ per pixel. Increasing the spatial resolution shifts the measured data further up along the KSrelation as long the resolution is coarser than the scale of single molecular clouds, where observations report a steepening of the star formation relation (Gutermuth et al. 2011; Willis et al. 2015). In the isolated galaxy and the early merger phases, the uniform gas distribution results in unaffected values for $\Sigma_{\text {gas }}$ while increasing resolution helps in separating regions with and without star formation.

\subsection{Identification of stellar clusters}

We use the built-in friends-of-friends (FoF) and SuBFIND (Springel et al. 2001; Dolag et al. 2009) algorithms of GADGET-3 to identify bound stellar clusters during the simulations. The FoF group finding and the SuBFIND-unbinding are performed only for new stellar particles formed during the simulations. In detail, SubFind differentiates all bound clusters in each snapshot by methodically examining all density peaks even if they are embedded in a more extended object. Our method differs from observational techniques in that we use the full 3D data available from the simulation, while observational data reduction often suffers from the loss of information due to projection effects. We however chose to employ SubFind for our cluster identification, as one of our goals is to study the build-up and merging of the young clusters, which might at different stages of their evolution be even temporarily embedded within each other, especially when seen in projection.

In defining a stellar cluster, we use a minimum particle number of 50, which translates into a minimum cluster mass of $M_{\mathrm{cl}, \min } \sim 200 M_{\odot}$ at our resolution. Our choice is somewhat more conservative compared to e.g. the definition of a minimum of 35 stars for a star cluster in Lada \& Lada (2003). On the other hand, observational samples of stellar clusters outside of the Milky Way are typically only complete above a few hundred $M_{\odot}$ in the M31 (Johnson et al. 2015) and the Magellanic clouds (Hunter et al. 2003) and a few $10^{3} M_{\odot}$ outside of the Local Group (Cook et al. 2012; Messa et al. 2018b). When studying the individual clusters, we will consider the entire simulated sample, whereas when fitting the cluster mass functions we will restrict the data in correspondence to the observed completeness limits.

An overview of the central cluster formation environment is shown in Fig. 4, in a 800 pc box centered on the most massive cluster formation region. The snapshot shown here is from the onset of the starburst at a simulation time of $t=160 \mathrm{Myr}$, when the destructive feedback from the young stellar population has not yet destroyed the gaseous structures in the star-forming central region (see Fig. 2). The left and rightmost panels in the top and middle row of Fig. 4 show the distribution of stars and gas in two $80 \mathrm{pc}$ zooms around the most massive cluster formation regions, which will give birth to the GC-like massive clusters. Stellar feedback in the leftmost panel of Fig. 4 has already started to form a cavity around the young stellar clusters, whereas the rightmost panel shows smaller mass clusters still embedded in the gaseous filament in which they form.

The left and rightmost panels in the bottom row of Fig. 4 show with separate colors the stellar particles bound to each individual cluster identified by SubFIND from the 80 pc slices in the top row. Most of the stellar particles in these two star-forming regions not bound to smaller objects are considered bound to the most massive cluster in each region, which is also why the masses and formation rates reported here are upper limits. Many of the smaller mass proto-clusters, each with their own bound population of stars, seem embedded in the massive clusters at least seen in projection. Some of these low mass clusters will coalesce with the more massive clusters, some will get disrupted, and the rest will remain as young individual star clusters. Plenty of clearly separate smaller mass clusters are already present, especially in the beads-on-a-string structures such as the bottom-left region of the rightmost column of Fig. 4.

The middle panel of the bottom row of Fig. 4 shows the center of mass of each cluster in the central region (top middle panel). The spatial distribution of clusters is mostly filamentary both on large and small scales, where the star and cluster formation follows the structure of the gas distribution.

\subsection{Cluster formation rate}

The evolution of the merger can be divided into three phases where the increase in star formation and the mass in young stellar clusters trace each other. In Fig. 5 we show the SFRs of the merger and the isolated dwarf compared to the cluster formation rates (CFRs) in both simulations defined as

$$
\mathrm{CFR}=\frac{M_{*, \mathrm{cl}}(\tau<10 \mathrm{Myr})}{10 \mathrm{Myr}}
$$

where $\tau$ is the mean stellar age and $M_{*, \text { cl }}(\tau<10 \mathrm{Myr})$ is the stellar mass in clusters with $\tau<10 \mathrm{Myr}$. We chose quite a short timescale of 10 Myr for our analysis, compared to e.g. studies of quiescent disk galaxies such as in Johnson et al. (2016), as the changes in the SFR and the subsequent CFR during the merger occur quite 


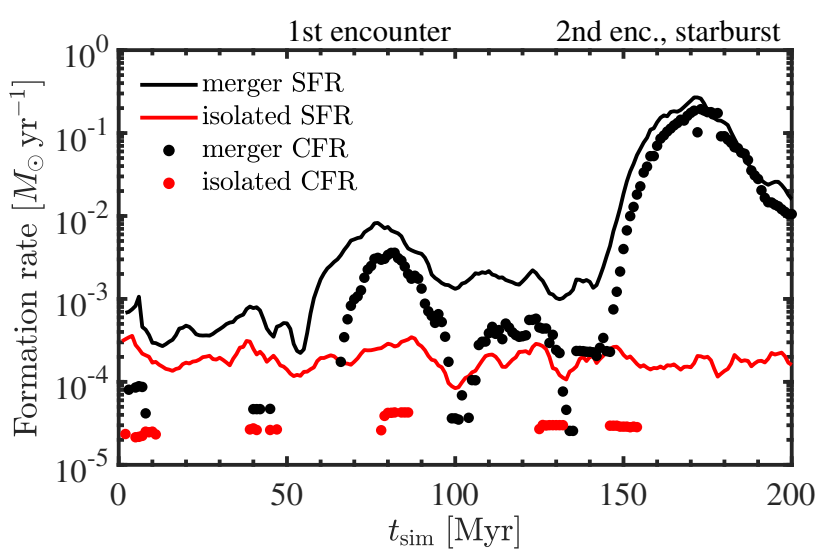

Figure 5. The total star formation rates of the merger and the isolated dwarf galaxy calculated for each snapshot from stellar particles with ages less than 5 Myr. The SFR is compared to the upper limit of the cluster formation rates, computed from clusters younger than $10 \mathrm{Myr}$. The snapshots with no young clusters have been omitted, which results in gaps in the data, especially for the isolated dwarf.

rapidly. In contrast to observational CFR results where the rates are lower limits, the CFR presented in Fig. 5 is an upper limit since we include here all the cluster data obtained from the simulation without corrections for observational bias.

As defined in Eq. 2, each cluster appears in the CFR data while it has a mean age less than the threshold age of $10 \mathrm{Myr}$ and a mass above the lower mass limit of $\sim 200 M_{\odot}$. The quiescent phase of star formation during the approach phase and in the isolated dwarf is accompanied by an episodic CFR where new low mass clusters form in a 40-50 Myr cycle.

In the merger simulation the cluster formation is more continuous. The CFR along the merger increases after the first pericenteric passage, but only up to half of the respective SFR. Most of the stars form outside of the few tens of bound clusters before the second passage at 160 Myr. After the second passage the mode of star formation abruptly changes, as now unlike in the quiescent phase and during the first passage, most of the star formation takes place in the cluster forming regions of the central high pressure environment (see Fig. 2). By simulation time $200 \mathrm{Myr}, 70 \%$ of all the stars formed have ended up in bound clusters, which corresponds to $7.8 \%$ of all the stellar mass in the system.

\subsection{Maximum cluster mass}

The maximum mass of the most massive stellar cluster is thought to be set by the combined disruption from shear forces and stellar feedback, with the final mass depending on the formation environment (ReinaCampos \& Kruijssen 2017). Dwarf galaxies and inter-

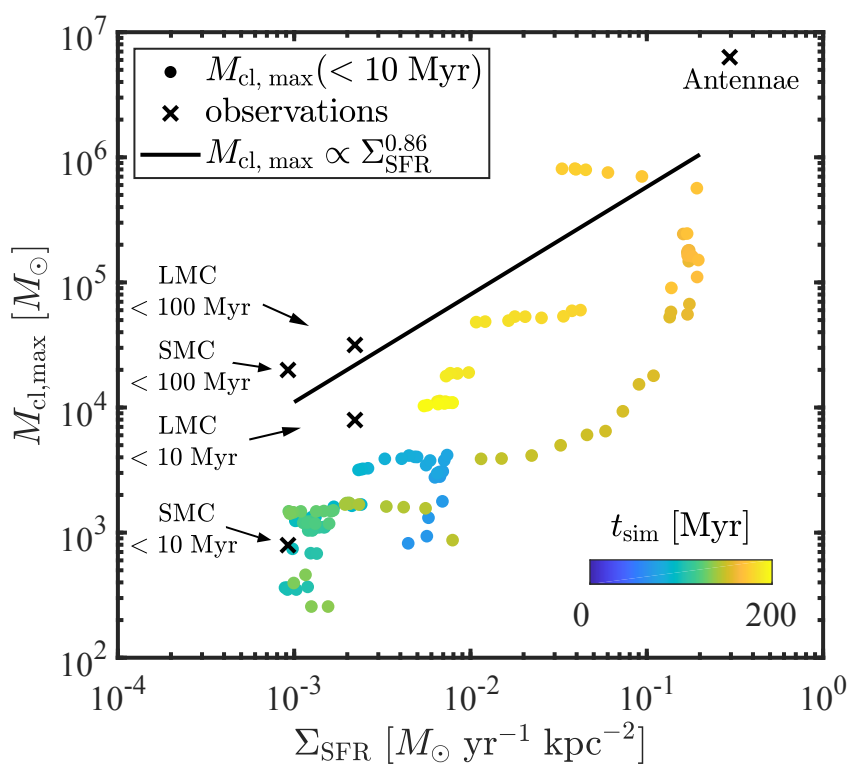

Figure 6. The mass of the most massive young cluster $(<$ $10 \mathrm{Myr}$ ) as a function of the SFR surface density, calculated in pixels with $\mathrm{SF}$ at resolution of $100 \mathrm{pc}$ per pixel. The black crosses show observed values for the most massive young clusters in the LMC and SMC (two age bins labeled on the left, Hunter et al. 2003) and in the Antennae merger (cluster age of the order of $1 \mathrm{Myr}$, Whitmore et al. 2010) where the

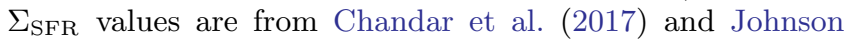
et al. (2017) respectively. The black diagonal line shows a model relation from Elmegreen (2018).

acting galaxies, with weak rotational shear, are found to harbor the most massive young stellar clusters (Weidner et al. 2010).

As the first step in connecting the cluster formation to the SF we show in Fig. 6 the most massive young $(<10$ Myr) cluster as a function of the mean star formation rate surface density $\left(\Sigma_{\mathrm{SFR}}\right)$ in $100 \mathrm{pc}$ pixels with ongoing star formation, in each snapshot along the entire merger. The maximum cluster mass follows the evolution of the SFR surface density. We compare our results to two age groups in the LMC and SMC (Hunter et al. 2003; Chandar et al. 2017), and to the observed most massive super star cluster in the Antennae merger (Whitmore et al. 2010; Johnson et al. 2017). The age group of massive clusters younger than $10 \mathrm{Myr}$ in the LMC and SMC agree well with our clusters formed during the first and second passages. The older age groups in the Magellanic clouds may on the other hand represent a different SF environment present at the time of their formation, uncorrelated with the present-day $\Sigma_{\mathrm{SFR}}$, thus showing unsurprisingly worse agreement with our results. The most massive clusters forming during the starburst fall along a trend between the young clusters in the Magellanic clouds and the upper extreme set by the Antennae. 

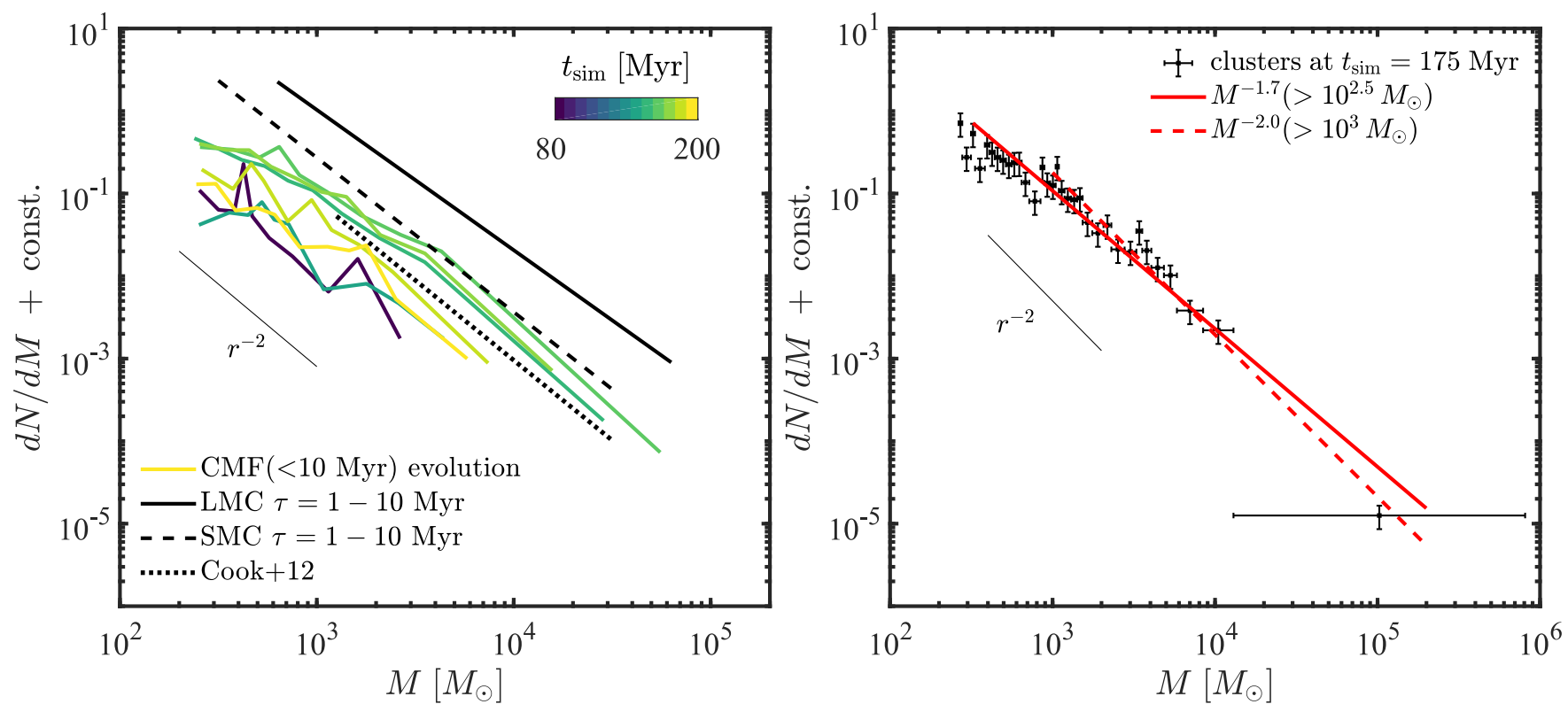

Figure 7. Left panel: the CMF of young $(<10 \mathrm{Myr})$ clusters after first passage at $80 \mathrm{Myr}$ and from the second passage until the merger (150-200 Myr) in 10 Myr steps. The clusters in each snapshot are binned into 10 bins with roughly equal number of clusters in each bin, omitting snapshots with less than 2 clusters per bin. The CMFs in the LMC (solid line) and SMC (dashed line) for clusters with ages between 1-10 Myr are from Fall \& Chandar (2012) and the ANGST survey CMF (dotted line) including clusters with ages up to $63 \mathrm{Myr}$ is from Cook et al. (2012). Right panel: same as on the left, immediately after the merger at time 175 Myr for maximum population size, now binned with 10 clusters per bin and showing the Poisson errors. The best fit CMF to clusters above $10^{2.5} M_{\odot}$ and $10^{3} M_{\odot}$ correspond to power-laws with indices $\alpha=-1.67 \pm 0.15$ and $\alpha=-1.96 \pm 0.18$, respectively.

We also compare to a model relation for self-gravitating clouds from Elmegreen (2018), which shows a slope similar $(\beta=0.86)$ to our trend.

\section{THE POPULATION OF STAR CLUSTERS}

\subsection{Cluster mass function}

The mass function (MF) in terms of the number of objects $N$ per mass bin $d M$ for both interstellar molecular clouds and stellar clusters can be described by a power-law $d N / d M \propto M^{\alpha}$ with a power-law index of $\alpha$. Observationally, molecular clouds have been shown to agree with a power-law index of $\alpha \sim-1.7--1.8$ (Lada et al. 1991; Heithausen et al. 1998; Kramer et al. 1998). Similarly, the cluster mass function (CMF) has been observed with a slightly steeper power-law index, closer to $\alpha \sim-2$ (see e.g. Lada \& Lada 2003 and Portegies Zwart et al. 2010 and references therein).

One of the strong points of our simulation comes from the combination of detailed subresolution models with high spatial and particle mass resolution. As a result we are able to follow the cloud collapse and the subsequent formation of stellar clusters in a wide mass range from a few $100 M_{\odot}$ to $10^{6} M_{\odot}$ during the entire interaction sequence. The evolution of the binned CMF calculated from young clusters $(<10 \mathrm{Myr})$ is given in the left panel of Fig. 7 in the dwarf galaxy merger after the first pericenter at $80 \mathrm{Myr}$ and in $10 \mathrm{Myr}$ intervals from 150 Myr onward. The cluster masses have been binned with equal number of clusters per bin, adaptively for each snapshot so that there are always ten bins per snapshot. The overall number of clusters in all of the mass bins increases while the slope of the CMF is established already after the first passage and remains quite constant as the merger proceeds. As shown in Fig. 6, the mass of the most massive young cluster increases with increasing $\Sigma_{\mathrm{SFR}}$, as the CMF shifts upward. The upper limit of the CMF and the hierarchical mass distribution towards lower masses is therefore simply set by the star formation environment, as discussed e.g. in Kruijssen (2015) and more recently in Mok et al. (2019) and Pfeffer et al. (2019).

The CMFs of the young clusters are compared in Fig. 7 against the power-law fits with slopes of $-1.7 \pm 0.02$ and $-1.87 \pm 0.25$ to young stellar clusters in the Large (LMC) and the Small Magellanic Clouds (SMC) respectively (Fall \& Chandar 2012). Fig. 7 includes also the CMF with a slope of $-1.94 \pm 0.26$ fit to clusters in dwarf galaxies from the ANGST project (Cook et al. 2012), fit above their completeness limit of $\sim 10^{3.5} M_{\odot}$ for clusters with ages up to $63 \mathrm{Myr}$. Our results for the simulated CMF shapes show good agreement with the observed CMFs. Note that the normalization of the CMFs de- 


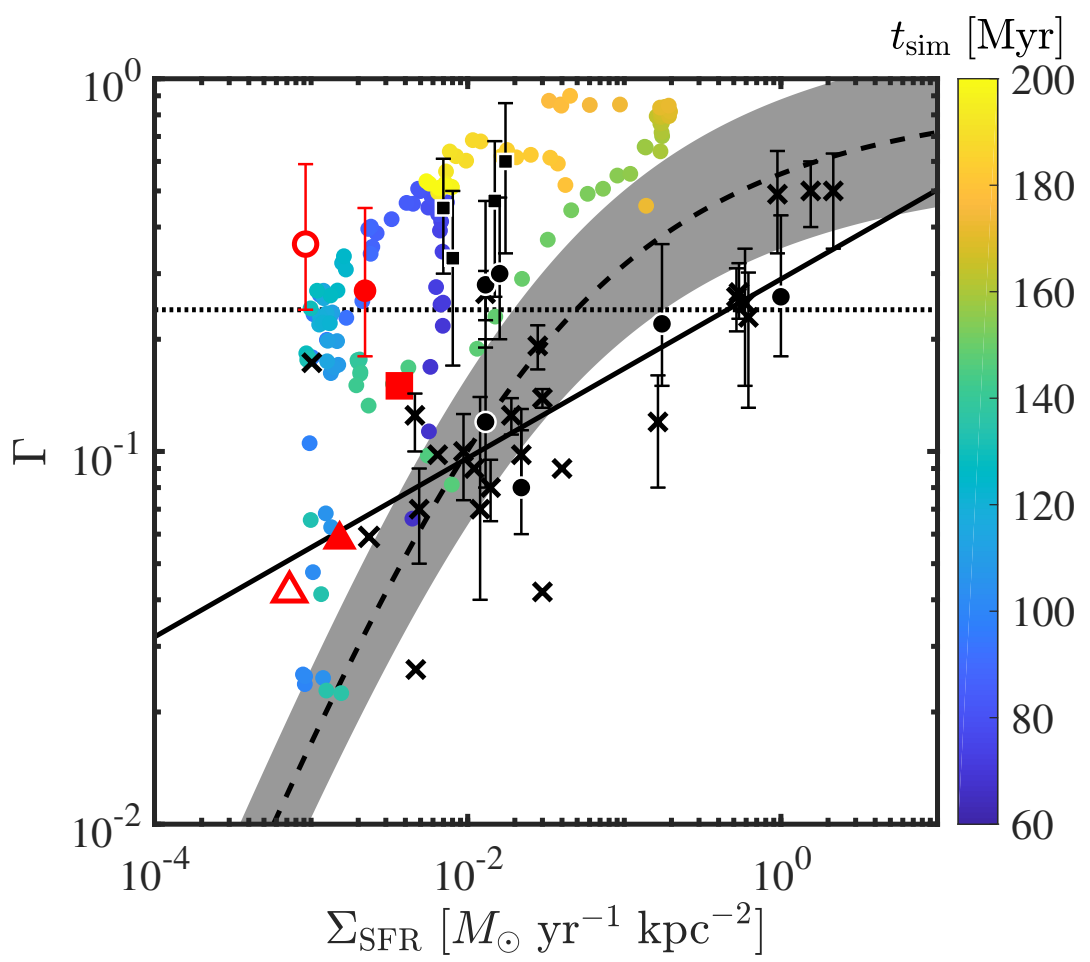

- LMC Goddard et al. 2010

- LMC Baumgardt et al. 2013

Ð LMC Chandar et al. 2017

$\Delta$ SMC Goddard et al. 2010

호 SMC Chandar et al. 2017

平 Johnson et al. 2016 tabulated

Fensch et al. 2019

Chandar et al. 2017

- Goddard et al. 2010 best fit

- - - Kruijssen 2012 model

. Chandar et al. 2017 constant

Figure 8. Cluster formation efficiency (Eq. 3) as a function of $\Sigma_{\text {SFR }}$ from the first pericentric passage onward. The $\Sigma_{\text {SFR }}$ has been calculated as the mean in pixels with SF, at pixel size of $100 \mathrm{pc}$. The solid line shows the observed relation from Goddard et al. (2010), the dashed line shows a model relation presented in Kruijssen (2012), with a \pm 0.2 dex region indicated in gray, and the dotted horizontal line is the constant $24 \%$ CFE found by Chandar et al. (2017). The red markers show observed values for the LMC (Goddard et al. 2010; Baumgardt et al. 2013; Chandar et al. 2017) and the SMC (Goddard et al. 2010; Chandar et al. 2017). The black squares with errorbars are from Fensch et al. (2019), the black circles from Chandar et al. (2017) and the crosses collected from Goddard et al. (2010), Adamo et al. (2011), Silva-Villa \& Larsen (2011), Annibali et al. (2011), Cook et al. (2012), Ryon et al. (2014), Lim \& Lee (2015), Adamo et al. (2015) and Johnson et al. (2016). Errors for the observed points without errorbars are either unavailable or smaller than the marker size.

pends on the total number of clusters in each system and is therefore arbitrary.

In the right hand panel of Fig. 7 we also show the CMF during the starburst at $t \sim 175 \mathrm{Myr}$, when the young clusters are numerous, binned with 10 clusters per bin. We fit a power-law with best-fit indices of $\alpha=$ $-1.67 \pm 0.15$ and $\alpha=-1.96 \pm 0.18$ to clusters above masses $10^{2.5} M_{\odot}$ and $10^{3} M_{\odot}$, respectively, to mimic the typical observational sample completeness limits. The fitted power-law indices agree well with the observed slopes in e.g. the Magellanic clouds (Fall \& Chandar 2012). The largest mass-bin includes the most massive globular cluster studied in L19.

\subsection{Cluster formation efficiency}

To further quantify the relationship between star formation and bound cluster formation, we calculate the cluster formation efficiency (CFE or $\Gamma$, Bastian 2008) as a function of time for which we use the form

$$
\Gamma=\frac{M_{*, \mathrm{cl}}(\tau<10 \mathrm{Myr})}{M_{*}(\tau<10 \mathrm{Myr})}
$$

Here $M_{*}(\tau<10 \mathrm{Myr})$ again includes all stellar mass formed during the last $10 \mathrm{Myr}$ and $M_{*, \mathrm{cl}}(\tau<10 \mathrm{Myr})$ includes the stellar mass in clusters with mean stellar ages less than 10 Myr. We show in Fig. 8 the CFE as a function of the $\Sigma_{\mathrm{SFR}}$ calculated from $100 \mathrm{pc}$ pixels with $\mathrm{SF}$. The CFE during the simulation is shown in Fig. 8 from the first pericentric passage onward, compared to various observed results and analytical predictions. For clarity, we omit from Fig. 8 the approach phase and the isolated dwarf as there are only very few clusters forming at any given time, at 10-20\% efficiency.

The results in Fig. 8 for the simulated CFE follow a similar trend with $\Sigma_{\mathrm{SFR}}$ as has been predicted analytically for e.g. a typical disk galaxy model in Kruijssen (2012). Observations of the CFE in a number of extra-galactic systems are shown in Fig. 8 from a set of references and the results for the Magellanic clouds are highlighted with red symbols. There is some uncertainty in the literature on how the clusters for determining the $\mathrm{CFE}$ are selected, which is reflected in e.g. the order of magnitude variance in the observed results for the Magellanic clouds shown in Fig. 8. When older 
clusters for example with ages between 10-100 Myr are included, the resulting CFE ends up lower due to the environment-dependent cluster destruction which may proceed as steeply as 1/age (Fall et al. 2005) given the presence of disrupting objects such as giant molecular clouds in a gas-rich environment (Gieles et al. 2006). Our results are derived from very young clusters principally to follow the varying SFR, which is also the suggested method in e.g. Chandar et al. (2017). In addition, we focus here on the population of young clusters as we do not detect significant cluster destruction during the duration of our simulation. However, it is unclear whether our stronger than observed cluster survival rate is due to the dwarf galaxy environment or the numerical implementation. Finally, one has to bear in mind that we only consider bound objects, whereas in observations young objects may also include open clusters and/or associations that are not bound.

The CFE during and after the first pericenter agrees with e.g. the results for the Magellanic clouds, which are interacting with the Milky Way and each other, somewhat reminiscent to the early phases of our simulation. During the merger, the CFE reaches 80-90\% for the duration of a few Myr, when the most massive clusters are forming and assembling hierarchically. We emphasize that our results for the CFE are absolute upper limits for the formation of bound stellar structures, as our cluster mass is obtained with SuBFIND from 3D data unavailable to observers. The low-mass, high gas-fraction system under study is however an analogue for high-redshift galaxy formation, and high values similar to our CFE in the early Universe have also been reported at least in numerical simulations (e.g. Li et al. 2017).

\subsection{Size and surface density}

In the left hand panel of Fig. 9 we show the mass and stellar half-mass radius of each of the simulated clusters at a simulation time of $t=175 \mathrm{Myr}$, separating the young clusters (darker red symbols) from the older cluster population (lighter red symbols). The simulated clusters are compared to the half-light radii and bestfit masses of clusters in the LMC, SMC, Fornax dwarf spheroidal and the Milky Way, obtained from McLaughlin \& van der Marel (2005), the best-fit effective radii and masses of the M31 clusters from the PHAT survey from Fouesneau et al. (2014) and Johnson et al. (2012), and the effective radii of clusters within two LEGUS galaxies (Ryon et al. 2017). In the McLaughlin \& van der Marel (2005) data the observed half-light radii have been obtained by fitting an EFF-profile (Elson et al. 1987) to the surface brightness profile while the simulated radii are taken directly from the particle data. For the LEGUS galaxies the effective radii are from the best-fit power-law surface brightness parameters obtained with GALFIT (Peng et al. 2002), considering only clusters with light profile power-law indices larger than 1.3. The M31 cluster radii have been obtained by interpolating the flux profiles.

Accurate radii for stellar clusters are often obtained through fitting the surface brightness or star count profile with a radial profile, which is why the left hand panel of Fig. 9 only includes fairly massive clusters. To assess the low mass clusters, the top right panel of Fig. 9 shows the total diameters of our clusters ${ }^{1}$, compared to the diameters ${ }^{2}$ of open clusters (OCs) in the Milky Way from van den Bergh (2006), based on data from Dias \& Lépine (2005).

The sizes of the massive clusters above a few $10^{3} M_{\odot}$ agree fairly well with the observed $M_{*}-r_{1 / 2}$ distribution, especially when we look at the young observed population. Similar cluster sizes of $1-10 \mathrm{pc}$ at masses above $10^{4} M_{\odot}$ have also been found in a high-resolution cluster formation study by Renaud et al. (2015). The lower mass clusters seem to be somewhat more compact than their observed counterparts, although the total sizes are in a slightly better agreement with the observed low mass clusters in the top right panel. The small sizes of the low-mass objects might be connected to the employed star formation scheme and will be investigated in greater detail in future work. For some of the smallest clusters the cluster radii are also close to the gravitational softening length. For such clusters the internal structure is not fully resolved and thus there is some uncertainty in the cluster sizes, because the gravitational motions, although still energy and angular momentum conserving, are softened.

The bottom row of Fig. 9 shows the effective and total mean stellar surface densities from the data points in the top row, either directly from the dataset (WM, LMC, SMC, Fornax with errors) or calculated as $\Sigma_{1 / 2}=$ $M\left(2 \pi r_{1 / 2}^{2}\right)^{-1}$ and $\Sigma_{\text {tot }}=M\left(\pi\left(d_{\text {tot }} / 2\right)^{2}\right)^{-1}$. Our clusters agree again best with observed young intermediate mass clusters, while the slightly too small radii of the lowermass clusters translate into higher surface densities than observed.

There is a tendency for older clusters to have larger sizes and lower mean surface densities, similarly to what is observed in e.g. LMC clusters (Elson et al. 1989; Mackey \& Gilmore 2003; Ferraro et al. 2019). Suggested reasons for the dilution of the central densities include

\footnotetext{
1 Twice the radii including $90 \%$ of the stellar mass

2 Maximum extent a cluster is separable from the background noise
} 

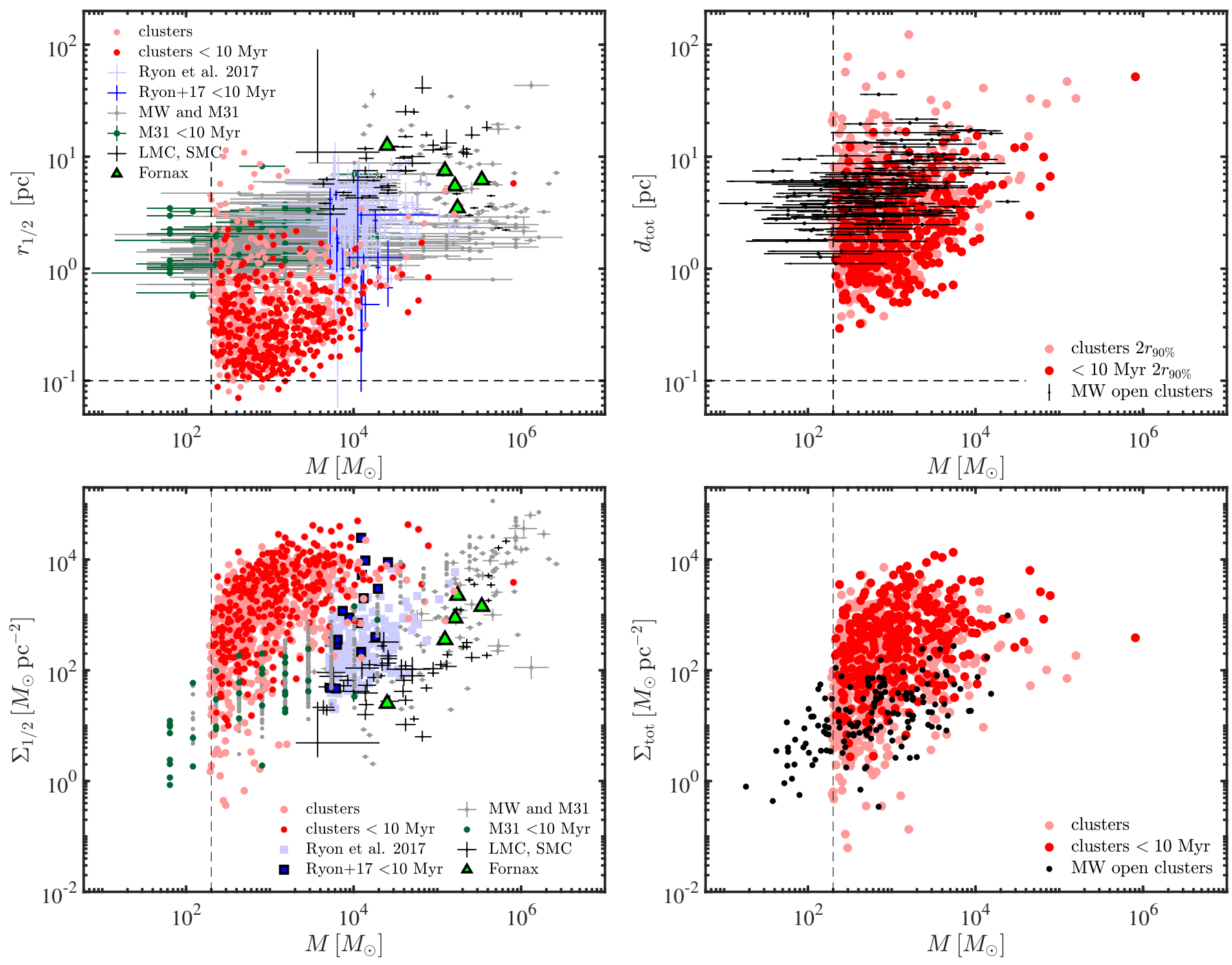

Figure 9. Top left: the stellar half mass radius for all (light red) and young (dark red) clusters with respect to stellar mass at simulation time 175 Myr. The masses and half-light radii for massive clusters in MW, LMC, SMC and the Fornax dwarf spheroidal are from McLaughlin \& van der Marel (2005), for M31 in the PHAT survey from Fouesneau et al. (2014) (masses) Johnson et al. (2012) (radii), and for clusters in two LEGUS galaxies from Ryon et al. (2017). The minimum cluster mass used in Subfind and the softening length of $0.1 \mathrm{pc}$ are indicated with horizontal solid and vertical lines. Top right: diameter measured as two times the radius including $90 \%$ of the cluster mass, compared to OCs in the Milky Way from van den Bergh (2006) (based on Dias \& Lépine 2005). Bottom left: stellar surface density within half-mass (half-light) radius for the data in top left panel. Bottom right: stellar surface density within the clusters for the data in top right panel.

internal stellar dynamics (Ferraro et al. 2019), varying tidal fields (Wilkinson et al. 2003) and binary black holes (Mackey et al. 2008). Our simulation includes a realistic IMF from $\sim 4 M_{\odot}$ upwards as well as stellar mass black holes in massive SNII remnants, but only resolved down to the gravitational softening length. A more detailed investigation into the dynamical evolution is beyond the scope of this article and will be addressed in future work.

\subsection{Gas content and age spread}

The star clusters form embedded within filamentary gas clouds, as indicated in Fig. 4. After a few Myr, the stellar feedback in the clusters expel the birth cloud, and the clusters become purely stellar, devoid of gas. Even though only less than $10 \%$ of the SNII energy released couples to the ISM (Walch \& Naab 2015), in L19 we found that at least in the massive clusters, the SNII feedback is easily able to deposit more than the binding energy in the gas with typical densities of $\sim 10^{3} M_{\odot} \mathrm{pc}^{-3}$ (see Fig. 3 in L19) when the SNII rates are up to 500 SNII per Myr per cluster.

The left panel in Fig. 10 shows the mass fraction of gas in each young ( $<10 \mathrm{Myr}$ ) cluster at a simulation time of $175 \mathrm{Myr}$ (as in the right panel of Fig. 7). The gas fraction has been calculated from gas residing within 

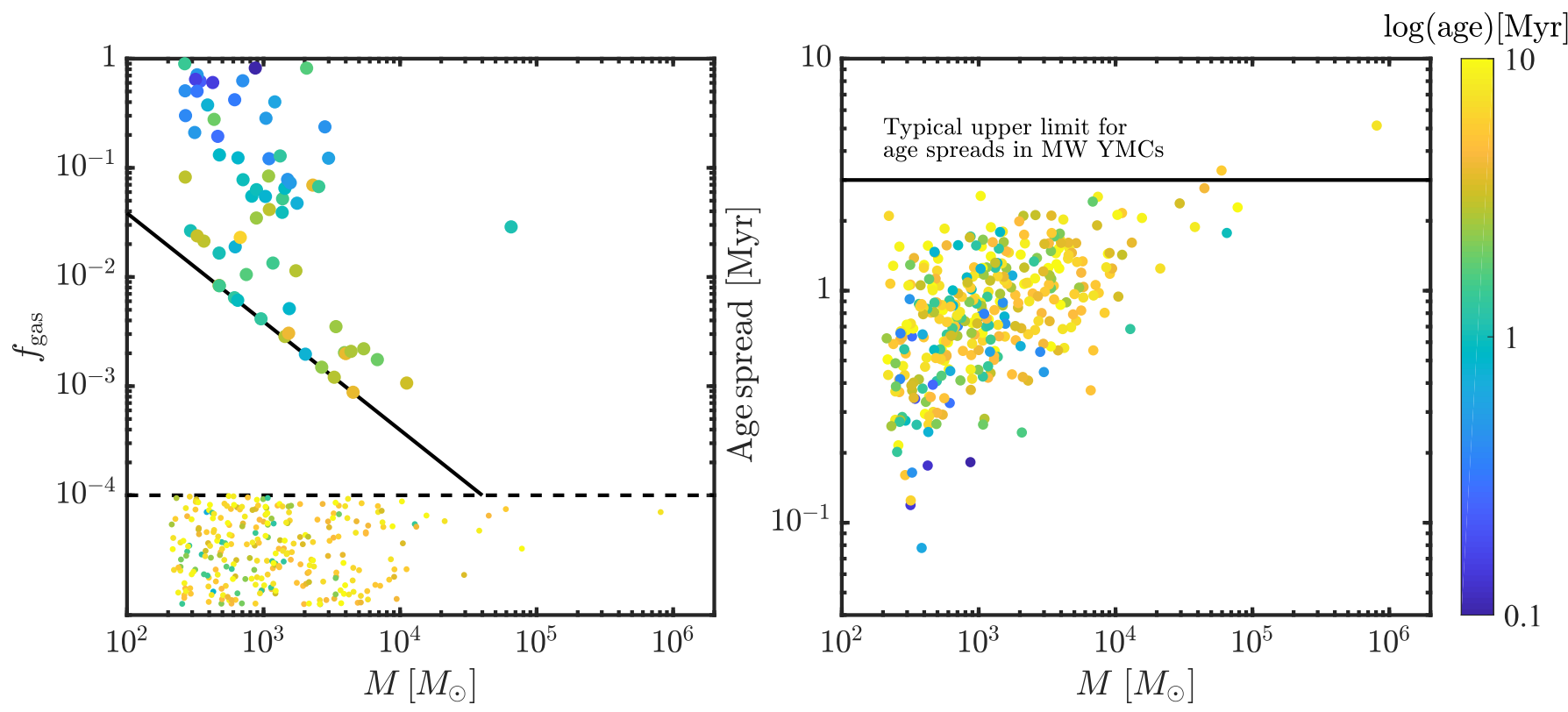

Figure 10. Left: Gas fraction, calculated from gas within two half-mass radii from the center of mass of all clusters younger than $10 \mathrm{Myr}$, at simulation time $175 \mathrm{Myr}$. The data points are colored by age. The black solid line shows the limit of one gas particle per cluster, and the randomly scattered points below $f_{\text {gas }}=10^{-4}$ are clusters with no gas. Right: The spread of ages within $1 \sigma$ on either side of the mean stellar age in the young clusters of the left panel. The horizontal line shows a typical upper limit for the age spread in YMCs in the Milky Way (Longmore et al. 2014).

two stellar half-mass radii of the center of mass of each cluster. Embedded clusters are considered as long as the bound stellar component is more than $\sim 200 M_{\odot}$ according to SuBFIND. The gas fractions are shown with respect to the stellar mass, color coded by mean stellar age. Clusters without gas have been spread in the bottom part of the $\mathrm{y}$-axis.

The youngest and least massive clusters are the most gas-rich, and at the age of a few Myr most of the clusters become gas-poor. This result shows how the gas expulsion takes place within the first few Myr of the cluster evolution. As the most extreme example and as also discussed in L19, once the most massive cluster begins to form, it expels all its gas by $7 \mathrm{Myr}$ into its formation. Stellar feedback before the type II supernovae lowers the total amount of energy required to unbind the gas, which results in gas expulsion fairly soon after the SNII feedback kicks in. Young stellar clusters are very rarely observed within their birth cloud after a few Myr (Lada \& Lada 2003; Bastian et al. 2014; Hollyhead et al. 2015; Hannon et al. 2019), in agreement with the general trend in Fig. 10.

Assuming a cluster forms from collapsing gas, the duration of the collapse and the time at which a cluster expels its gas set the primary limits for the formation time and age spread of each cluster. When inferring ages of observed clusters, single stellar population models are often used, assuming all stars formed coevally. However, the age distributions in some local stellar clusters have been quantified on a star by star basis (Longmore et al. 2014). Age spreads, when detected, are commonly reported as a few Myr (Bik et al. 2011; Da Rio et al. 2010), typically less than 3 Myr. For massive clusters in external galaxies, the age spread is expected to be no larger than a few 10 of Myrs (Bastian \& Lardo 2018).

We show the $1 \sigma$ spread in the ages of the stellar particles within the young simulated clusters in the right panel of Fig. 10 as a function of cluster mass and colorcoded by cluster age. The spread is here defined as the width of the distribution including $1 \sigma$ on either side of the mean stellar age. The clusters seem to form coevally; the age spread in the simulated clusters is typically in the range $\sim 0.1-2$ Myr with only a few clusters with an age spread above $2 \mathrm{Myr}$, well within the typical values observed in the Milky Way. Even the most massive cluster, which is built hierarchically, forms its stellar mass on the free-fall timescale, resulting in an age spread of 5.1 Myr. In other words, the small structures collapse almost simultaneously and merge afterwards. Similar results for stellar age spreads have been reported in other recent numerical studies probing e.g. the high-redshift environment of GC formation such as Li et al. (2017) and Ma et al. (2019). As in Fig. 10, Ma et al. (2019) report higher age spreads with increasing cluster mass, though they study masses above $10^{3.5} M_{\odot}$.

The derived age spreads are also related to the discussion regarding multiple stellar populations in massive star clusters. Even though we do not include processes 
necessary to assess the chemical anomalies typically used in the identification of the multiple stellar populations, we can draw some conclusions from the age distribution in the young clusters. Since the clusters become gaspoor at a relatively young age, large amounts of future star formation from enriched material is not supported by our results. Gas accreted from the ISM and expelled by stellar feedback or stellar mass loss could be used for future star formation (Conroy \& Spergel 2011), however all of our clusters include little or no gas $(<10 \%)$ in the $100 \mathrm{Myr}$ old merger remnant. On the other hand, the hierarchical formation of the clusters enables the first forming stars to enrich the infalling material for slightly later star formation during the formation phase. This would require a second enriched population, if present, to be enriched by some fairly rapid processes such as fast-rotating stars or massive binaries (Bastian \& Lardo 2018), rather than e.g. AGB-winds.

\section{CONCLUSIONS}

We have studied the formation of stellar clusters in a high-resolution simulation of a merger between two gasrich dwarf galaxies, concentrating on the observational properties of the young cluster population. The star formation properties, spanning three orders of magnitude in $\Sigma_{\mathrm{SFR}}$ during the simulation, were shown to agree with resolved observations of $\Sigma_{\mathrm{SFR}}-\Sigma_{\text {gas }}$ for galaxies in the present-day universe. The CFR and CFE along the merger correlate with the SFR and $\Sigma_{\mathrm{SFR}}$, respectively.

The $\Sigma_{\mathrm{SFR}}$ during the approach and the first pericentric passage result in similar values to the present-day Magellanic clouds. The young clusters, defined by a mean age of $10 \mathrm{Myr}$ or less, present also comparable values for the correlation between $\Sigma_{\mathrm{SFR}}$ and the most massive cluster in equivalent age interval in the Magellanic clouds. The CMF of the young SCs, which already has its shape after the first pericentric passage, is similar to a wide range of observed SCs in dwarf galaxies, also outside of the Local Group. The CMF of the young SCs retains its shape all the way through the simulation and the mass of the most massive young cluster increases almost linearly with $\Sigma_{\mathrm{SFR}}$. We fit a cluster mass function with a power-law index of the order of $\alpha \sim 2$ to the young SC population after the starburst.

A caveat of our simulations is that we do not detect significant amounts of cluster disruption after their formation, reported in massive galaxies such as the Milky Way and the Antennae (up to $90 \%$ per decade, e.g. Whitmore et al. 2007) or the Magellanic clouds (Chandar et al. 2010). Our clusters may form slightly too compact and therefore be more resistant to disruption (see Section 4.3). This potential caveat will be addressed in future work using modified star formation prescriptions and more detailed $N$-body dynamics.

Most of the stellar clusters at the massive end of the CMF, as those discussed in L19, have sizes, mean surface densities and stellar age spreads consistent with observed massive young clusters. There is also a slight tendency for older SCs to have larger half-mass radii and lower mean surface densities. The small mass clusters in the simulation seem to form too concentrated, and may therefore be resistant to infant mortality or cluster destruction. In future work we will investigate what is needed to obtain a more realistic size distribution. We will also inspect whether the small sizes are connected to the low cluster disruption rate, which may also result from the dwarf galaxy environment itself. The stellar age spreads in the young SCs are typically between 0.1 and $2 \mathrm{Myr}$. The largest spread of 5.1 Myr is found in the most massive cluster, which forms hierarchically from tens of smaller clusters along the CMF.

The merger scenario investigated here resulted in the formation of a stellar cluster population with a mass range from GC-like objects $\left(8 \times 10^{5} M_{\odot}\right)$ down to a few hundred $M_{\odot}$. The low shear and shallow gravitational potential of the system consisting of low mass dwarf galaxies enable long life times and easy accretion for even the smaller mass range of SCs. The formation of SCs in interacting dwarf galaxies presents therefore a compelling means for e.g. the origin of ex-situ SCs in outer halos of massive galaxies.

This study, which is part of the GRIFFIN project aimed at resolving the impact of individual massive stars on galaxy evolution, demonstrates that it is now possible to investigate the star cluster populations of entire galaxies, and therefore also the effect of clustered supernova explosions on galaxy evolution, with novel highresolution numerical simulations. This approach is a challenge for galaxy evolution modelling (Naab \& Ostriker 2017) and will hopefully result in novel insights on the underlying processes regulating galaxy evolution.

N.L. acknowledges the financial support by the Jenny and Antti Wihuri Foundation. T.N. acknowledges support from the DFG cluster of excellence "ORIGINS". N.L. and P.H.J. acknowledge support by the European Research Council via ERC Consolidator Grant KETJU (no. 818930). C.-Y.H. acknowledges The Center for Computational Astrophysics, supported by the Simons Foundation. S.W. acknowledges support by the European Research Council via ERC Starting Grant RADFEEDBACK (no. 679852) and by the German Science Foundation via CRC956, Project C5. U.P.S. and B.P.M. acknowledge support by an Emmy Noether grant of the Deutsche Forschungsgemeinschaft (DFG, German Research Foundation) under the project number MO 2979/1-1. The computations were carried out at CSC - IT Center for 
Science Ltd. in Finland and at Max-Planck Institute for Astrophysics in Germany.

\section{REFERENCES}

Adamo, A., Kruijssen, J. M. D., Bastian, N., Silva-Villa, E., \& Ryon, J. 2015, MNRAS, 452, 246

Adamo, A., Östlin, G., \& Zackrisson, E. 2011, MNRAS, 417, 1904

Annibali, F., Tosi, M., Aloisi, A., \& van der Marel, R. P. 2011, AJ, 142, 129

Arata, S., Yajima, H., \& Nagamine, K. 2018, MNRAS, 475, 4252

Bastian, N. 2008, MNRAS, 390, 759

Bastian, N., Covey, K. R., \& Meyer, M. R. 2010, ARA\&A, 48, 339

Bastian, N., Gieles, M., Lamers, H. J. G. L. M., Scheepmaker, R. A., \& de Grijs, R. 2005, A\&A, 431, 905

Bastian, N., Hollyhead, K., \& Cabrera-Ziri, I. 2014, MNRAS, 445, 378

Bastian, N., \& Lardo, C. 2018, ARA\&A, 56, 83

Bastian, N., Schweizer, F., Goudfrooij, P., Larsen, S. S., \& Kissler-Patig, M. 2013, MNRAS, 431, 1252

Baumgardt, H., Parmentier, G., Anders, P., \& Grebel, E. K. 2013, MNRAS, 430, 676

Bekki, K., Forbes, D. A., Beasley, M. A., \& Couch, W. J. 2002, MNRAS, 335, 1176

Bekki, K., Yahagi, H., Nagashima, M., \& Forbes, D. A. 2008, MNRAS, 387, 1131

Bik, A., Henning, T., Stolte, A., et al. 2011, The Astrophysical Journal, 744, 87. https: //doi.org/10.1088\%2F0004-637x\%2F744\%2F2\%2F87

Bisbas, T. G., Haworth, T. J., Williams, R. J. R., et al. 2015, MNRAS, 453, 1324

Bonnell, I. A., Bate, M. R., \& Vine, S. G. 2003, MNRAS, 343,413

Bothwell, M. S., Kennicutt, R. C., \& Lee, J. C. 2009, MNRAS, 400, 154

Bournaud, F., Duc, P.-A., \& Emsellem, E. 2008, MNRAS, 389, L8

Bullock, J. S., Dekel, A., Kolatt, T. S., et al. 2001, ApJ, 555,240

Chandar, R., Fall, S. M., \& Whitmore, B. C. 2010, ApJ, 711,1263

-. 2015, ApJ, 810, 1

Chandar, R., Fall, S. M., Whitmore, B. C., \& Mulia, A. J. 2017, ApJ, 849, 128

Chieffi, A., \& Limongi, M. 2004, ApJ, 608, 405

Choksi, N., \& Gnedin, O. Y. 2019, MNRAS, 486, 331

Choksi, N., Gnedin, O. Y., \& Li, H. 2018, MNRAS, 480, 2343
Clark, P. C., Glover, S. C. O., \& Klessen, R. S. 2012, MNRAS, 420, 745

Conroy, C., \& Spergel, D. N. 2011, ApJ, 726, 36

Cook, D. O., Seth, A. C., Dale, D. A., et al. 2012, ApJ, 751,100

Cottaar, M., Meyer, M. R., Andersen, M., \& Espinoza, P. 2012, A\&A, 539, A5

Cullen, L., \& Dehnen, W. 2010, MNRAS, 408, 669

Da Rio, N., Robberto, M., Soderblom, D. R., et al. 2010, ApJ, 722, 1092

Dehnen, W., \& Aly, H. 2012, MNRAS, 425, 1068

Dias, W. S., \& Lépine, J. R. D. 2005, ApJ, 629, 825

Dolag, K., Borgani, S., Murante, G., \& Springel, V. 2009, MNRAS, 399, 497

El-Badry, K., Quataert, E., Weisz, D. R., Choksi, N., \& Boylan-Kolchin, M. 2019, MNRAS, 482, 4528

Ellison, S. L., Mendel, J. T., Patton, D. R., \& Scudder, J. M. 2013, MNRAS, 435, 3627

Elmegreen, B. G. 2008, ApJ, 672, 1006

—. 2018, ApJ, 869, 119

Elmegreen, B. G., \& Efremov, Y. N. 1996, ApJ, 466, 802

—. 1997, ApJ, 480, 235

Elmegreen, B. G., \& Falgarone, E. 1996, ApJ, 471, 816

Elson, R. A. W., Fall, S. M., \& Freeman, K. C. 1987, ApJ, 323,54

Elson, R. A. W., Freeman, K. C., \& Lauer, T. R. 1989, ApJL, 347, L69

Emerick, A., Bryan, G. L., \& Mac Low, M.-M. 2018, ApJL, $865, \mathrm{~L} 22$

Fall, S. M., \& Chandar, R. 2012, ApJ, 752, 96

Fall, S. M., Chandar, R., \& Whitmore, B. C. 2005, ApJL, 631, L133

Fall, S. M., Krumholz, M. R., \& Matzner, C. D. 2010, ApJL, 710, L142

Fall, S. M., \& Zhang, Q. 2001, ApJ, 561, 751

Fensch, J., Duc, P.-A., Boquien, M., et al. 2019, A\&A, 628, A60

Ferraro, F. R., Lanzoni, B., Dalessandro, E., et al. 2019, arXiv e-prints, arXiv:1909.02049

Fouesneau, M., Johnson, L. C., Weisz, D. R., et al. 2014, ApJ, 786, 117

Gatto, A., Walch, S., Low, M.-M. M., et al. 2015, MNRAS, 449, 1057

Genzel, R., Eisenhauer, F., \& Gillessen, S. 2010, Reviews of Modern Physics, 82, 3121 
Georgy, C., Ekström, S., Eggenberger, P., et al. 2013, A\&A, 558, A103

Gieles, M., Portegies Zwart, S. F., Baumgardt, H., et al. 2006, MNRAS, 371, 793

Gingold, R. A., \& Monaghan, J. J. 1977, MNRAS, 181, 375

Girichidis, P., Walch, S., Naab, T., et al. 2016, MNRAS, 456, 3432

Glover, S. C. O., \& Clark, P. C. 2012, MNRAS, 421, 116

Glover, S. C. O., \& Mac Low, M.-M. 2007, ApJS, 169, 239

Goddard, Q. E., Bastian, N., \& Kennicutt, R. C. 2010, MNRAS, 405, 857

Górski, K. M., \& Hivon, E. 2011, HEALPix: Hierarchical Equal Area isoLatitude Pixelization of a sphere, Astrophysics Source Code Library, , , ascl:1107.018

Grasha, K., Calzetti, D., Adamo, A., et al. 2017, ApJ, 840, 113

Gutermuth, R. A., Pipher, J. L., Megeath, S. T., et al. 2011, ApJ, 739, 84

Haardt, F., \& Madau, P. 1996, ApJ, 461, 20

Halbesma, T. L. R., Grand, R. J. J., Gómez, F. A., et al. 2019, arXiv e-prints, arXiv:1909.02630

Hannon, S., Lee, J. C., Whitmore, B. C., et al. 2019, MNRAS, 490, 4648

Harris, W. E. 1991, ARA\&A, 29, 543

Heithausen, A., Bensch, F., Stutzki, J., Falgarone, E., \& Panis, J. F. 1998, A\&A, 331, L65

Hernquist, L. 1990, ApJ, 356, 359

Hollyhead, K., Bastian, N., Adamo, A., et al. 2015, MNRAS, 449, 1106

Hopkins, P. F. 2013, MNRAS, 428, 2840

Hopkins, P. F., Cox, T. J., Hernquist, L., et al. 2013, MNRAS, 430, 1901

Howard, C. S., Pudritz, R. E., \& Harris, W. E. 2018, Nat. Astron., 2, 725

Hu, C.-Y., Naab, T., Glover, S. C. O., Walch, S., \& Clark, P. C. 2017, MNRAS, 471, 2151

Hu, C.-Y., Naab, T., Walch, S., Glover, S. C. O., \& Clark, P. C. 2016, MNRAS, 458, 3528

Hu, C.-Y., Naab, T., Walch, S., Moster, B. P., \& Oser, L. 2014, MNRAS, 443, 1173

Huang, S., Haynes, M. P., Giovanelli, R., et al. 2012, AJ, 143,133

Hunter, D. A., Elmegreen, B. G., Dupuy, T. J., \& Mortonson, M. 2003, AJ, 126, 1836

Johnson, L. C., Seth, A. C., Dalcanton, J. J., et al. 2012, ApJ, 752, 95

-. 2015, ApJ, 802, 127

—. 2016, ApJ, 827, 33

—. 2017, ApJ, 839, 78

Karakas, A. I. 2010, MNRAS, 403, 1413
Kennicutt, Jr., R. C. 1998, ApJ, 498, 541

Kim, C.-G., \& Ostriker, E. C. 2018, ApJ, 853, 173

Kim, J.-h., Ma, X., Grudić, M. Y., et al. 2018, MNRAS, 474, 4232

Klessen, R. S. 2001, ApJ, 556, 837

Kramer, C., Stutzki, J., Rohrig, R., \& Corneliussen, U. 1998, A\&A, 329, 249

Kroupa, P. 2001, MNRAS, 322, 231

Kruijssen, J. M. D. 2012, MNRAS, 426, 3008

-. 2014, Classical and Quantum Gravity, 31, 244006

—. 2015, MNRAS, 454, 1658

Krumholz, M. R., \& McKee, C. F. 2019, arXiv e-prints, arXiv:1909.01565

Lada, C. J., \& Lada, E. A. 2003, ARA\&A, 41, 57

Lada, E. A., Bally, J., \& Stark, A. A. 1991, ApJ, 368, 432

Lahén, N., Johansson, P. H., Rantala, A., Naab, T., \& Frigo, M. 2018, MNRAS, 475, 3934

Lahén, N., Naab, T., Johansson, P. H., et al. 2019, ApJL, 879, L18

Larsen, S. S. 2004, A\&A, 416, 537

Lejeune, T., Cuisinier, F., \& Buser, R. 1997, A\&AS, 125, 229

-. 1998, A\&AS, 130, 65

Leroy, A. K., Walter, F., Brinks, E., et al. 2008, AJ, 136, 2782

Li, H., Gnedin, O. Y., Gnedin, N. Y., et al. 2017, ApJ, 834, 69

Li, H., Vogelsberger, M., Marinacci, F., \& Gnedin, O. Y. 2019, MNRAS, 487, 364

Li, Y., Mac Low, M.-M., \& Klessen, R. S. 2004, ApJL, 614, L29

-. 2005, ApJ, 626, 823

Lim, S., \& Lee, M. G. 2015, ApJ, 804, 123

Longmore, S. N., Kruijssen, J. M. D., Bastian, N., et al. 2014, Protostars and Planets VI, 291

Lucy, L. B. 1977, AJ, 82, 1013

Ma, X., Grudić, M. Y., Quataert, E., et al. 2019, arXiv e-prints, arXiv:1906.11261

Mackey, A. D., \& Gilmore, G. F. 2003, MNRAS, 338, 120

Mackey, A. D., Wilkinson, M. I., Davies, M. B., \& Gilmore, G. F. 2008, MNRAS, 386, 65

Mapelli, M. 2017, MNRAS, 467, 3255

McLaughlin, D. E., \& van der Marel, R. P. 2005, ApJS, 161,304

McMillan, S. L. W., Vesperini, E., \& Portegies Zwart, S. F. 2007, ApJL, 655, L45

Messa, M., Adamo, A., Calzetti, D., et al. 2018a, MNRAS, 477,1683

Messa, M., Adamo, A., Östlin, G., et al. 2018b, MNRAS, 473,996 
Meurer, G. R., Heckman, T. M., Leitherer, C., et al. 1995, AJ, 110, 2665

Mok, A., Chandar, R., \& Fall, S. M. 2019, ApJ, 872, 93

Naab, T., \& Ostriker, J. P. 2017, ARA\&A, 55, 59

Nelson, R. P., \& Langer, W. D. 1997, ApJ, 482, 796

O'Connell, R. W., Gallagher, III, J. S., \& Hunter, D. A. 1994, ApJ, 433, 65

Padoan, P., Haugbølle, T., Nordlund, A., \& Frimann, S. 2017, ApJ, 840, 48

Parker, R. J., \& Goodwin, S. P. 2007, MNRAS, 380, 1271

Paudel, S., Smith, R., Yoon, S. J., Calderón-Castillo, P., \& Duc, P.-A. 2018, ApJS, 237, 36

Pearson, S., Besla, G., Putman, M. E., et al. 2016, MNRAS, 459, 1827

Peng, C. Y., Ho, L. C., Impey, C. D., \& Rix, H.-W. 2002, AJ, 124, 266

Peters, T., Naab, T., Walch, S., et al. 2017, MNRAS, 466, 3293

Pfeffer, J., Bastian, N., Kruijssen, J. M. D., et al. 2019, MNRAS, 490, 1714

Pfeffer, J., Kruijssen, J. M. D., Crain, R. A., \& Bastian, N. 2018, MNRAS, 475, 4309

Portegies Zwart, S. F., McMillan, S. L. W., \& Gieles, M. 2010, ARA\&A, 48, 431

Price, D. J. 2008, Journal of Computational Physics, 227, 10040 . http://www.sciencedirect.com/science/article/ pii/S0021999108004270

Privon, G. C., Stierwalt, S., Patton, D. R., et al. 2017, ApJ, 846, 74

Read, J. I., Hayfield, T., \& Agertz, O. 2010, MNRAS, 405, 1513

Reina-Campos, M., \& Kruijssen, J. M. D. 2017, MNRAS, 469, 1282

Renaud, F. 2018, NewAR, 81, 1

Renaud, F., Agertz, O., \& Gieles, M. 2017, MNRAS, 465, 3622

Renaud, F., Bournaud, F., \& Duc, P.-A. 2015, MNRAS, 446, 2038

Renaud, F., Gieles, M., \& Boily, C. M. 2011, MNRAS, 418, 759

Rochau, B., Brandner, W., Stolte, A., et al. 2010, ApJL, 716, L90

Ryon, J. E., Adamo, A., Bastian, N., et al. 2014, AJ, 148, 33

Ryon, J. E., Gallagher, J. S., Smith, L. J., et al. 2017, ApJ, 841,92
Saitoh, T. R., Daisaka, H., Kokubo, E., et al. 2010, in Astronomical Society of the Pacific Conference Series, Vol. 423, Galaxy Wars: Stellar Populations and Star Formation in Interacting Galaxies, ed. B. Smith, J. Higdon, S. Higdon, \& N. Bastian, 185

Saitoh, T. R., \& Makino, J. 2013, ApJ, 768, 44

Salim, S., Rich, R. M., Charlot, S., et al. 2007, ApJS, 173, 267

Sanders, D. B., \& Mirabel, I. F. 1996, ARA\&A, 34, 749

Schiminovich, D., Wyder, T. K., Martin, D. C., et al. 2007, ApJS, 173, 315

Silva-Villa, E., \& Larsen, S. S. 2011, A\&A, 529, A25

Skinner, M. A., \& Ostriker, E. C. 2015, ApJ, 809, 187

Smith, B. J., Struck, C., Hancock, M., et al. 2007, AJ, 133, 791

Spitzer, L. 1978, Physical processes in the interstellar medium (Wiley-Interscience), doi:10.1002/9783527617722

Springel, V. 2005, MNRAS, 364, 1105

Springel, V., Di Matteo, T., \& Hernquist, L. 2005, MNRAS, 361, 776

Springel, V., White, S. D. M., Tormen, G., \& Kauffmann, G. 2001, MNRAS, 328, 726

Steinwandel, U. P., Moster, B. P., Naab, T., Hu, C.-Y., \& Walch, S. 2019, arXiv e-prints, arXiv:1907.13153

van den Bergh, S. 2006, AJ, 131, 1559

Vanzella, E., Calura, F., Meneghetti, M., et al. 2019, MNRAS, 483, 3618

Vázquez-Semadeni, E., González-Samaniego, A., \& Colín, P. 2017, MNRAS, 467, 1313

Walch, S., \& Naab, T. 2015, MNRAS, 451, 2757

Walch, S., Girichidis, P., Naab, T., et al. 2015, MNRAS, 454, 238

Wang, L., Spurzem, R., Aarseth, S., et al. 2016, MNRAS, 458, 1450

Ward, J. L., \& Kruijssen, J. M. D. 2018, MNRAS, 475, 5659

Weidner, C., Bonnell, I. A., \& Zinnecker, H. 2010, ApJ, 724, 1503

Westera, P., Lejeune, T., Buser, R., Cuisinier, F., \& Bruzual, G. 2002, A\&A, 381, 524

Whitmore, B. C., Chandar, R., \& Fall, S. M. 2007, AJ, 133, 1067

Whitmore, B. C., Zhang, Q., Leitherer, C., et al. 1999, AJ, 118, 1551

Whitmore, B. C., Chandar, R., Schweizer, F., et al. 2010, AJ, 140, 75

Whitmore, B. C., Brogan, C., Chandar, R., et al. 2014, ApJ, 795, 156

Wiersma, R. P. C., Schaye, J., \& Smith, B. D. 2009, MNRAS, 393, 99 
Wilkinson, M. I., Hurley, J. R., Mackey, A. D., Gilmore, G. F., \& Tout, C. A. 2003, MNRAS, 343, 1025
Willis, S., Guzman, A., Marengo, M., et al. 2015, ApJ, 809, 87

Zhang, Q., \& Fall, S. M. 1999, ApJL, 527, L81 